OPEN ACCESS

Edited by:

Armin Michael Nagel, University of Erlangen

Nuremberg, Germany

Reviewed by:

Peter Lundberg,

Linköping University, Sweden

Moritz Zaiss,

University Hospital Erlangen, Germany

${ }^{*}$ Correspondence:

Kristin P. O'Grady

kristin.p.ogrady@vumc.org

Specialty section: This article was submitted to Applied Neuroimaging, a section of the journal

Frontiers in Neurology

Received: 25 August 2021 Accepted: 01 February 2022 Published: 25 February 2022

Citation

O'Grady KP, Satish S, Owen QR, Box BA, Bagnato F, Combes AJE, Cook SR, Westervelt HJ, Feiler HR, Lawless RD, Sarma A, Malone SD,

Ndolo JM, Yoon K, Dortch RD,

Rogers BP and Smith SA (2022) Relaxation-Compensated Chemical Exchange Saturation Transfer MRI in the Brain at 7T: Application in Relapsing-Remitting Multiple Sclerosis. Front. Neurol. 13:764690.

doi: 10.3389/fneur.2022.764690

\section{Relaxation-Compensated Chemical Exchange Saturation Transfer MRI in the Brain at 7T: Application in Relapsing-Remitting Multiple Sclerosis}

Kristin P. O'Grady ${ }^{1,2 *}$, Sanjana Satish ${ }^{1}$, Quinn R. Owen ${ }^{1}$, Bailey A. Box ${ }^{1}$, Francesca Bagnato ${ }^{3,4}$, Anna J. E. Combes ${ }^{1,2}$, Sarah R. Cook ${ }^{1}$, Holly James Westervelt ${ }^{5}$, Haley R. Feiler ${ }^{1}$, Richard D. Lawless ${ }^{1}$, Asha Sarma ${ }^{2}$, Shekinah D. Malone ${ }^{6}$, Josephine M. Ndolo ${ }^{2}$, Keejin Yoon ${ }^{3}$, Richard D. Dortch ${ }^{7}$, Baxter P. Rogers ${ }^{1,2,8,9}$ and Seth A. Smith ${ }^{1,2,9}$

${ }^{1}$ Vanderbilt University Institute of Imaging Science, Vanderbilt University Medical Center, Nashville, TN, United States, ${ }^{2}$ Department of Radiology and Radiological Sciences, Vanderbilt University Medical Center, Nashville, TN, United States, ${ }^{3}$ Neuroimaging Unit, Division of Neuroimmunology, Department of Neurology, Vanderbilt University Medical Center, Nashville, TN, United States, ${ }^{4}$ Department of Neurology, Nashville VA Medical Center, TN Valley Healthcare System, Nashville, TN, United States, ${ }^{5}$ Division of Behavioral and Cognitive Neurology, Department of Neurology, Vanderbilt University Medical Center, Nashville, TN, United States, ${ }^{6}$ School of Medicine, Meharry Medical College, Nashville, TN, United States, ${ }^{7}$ Department of Translational Neuroscience, Barrow Neurological Institute, Phoenix, AZ, United States, ${ }^{8}$ Department of Psychiatry and Behavioral Sciences, Vanderbilt University Medical Center, Nashville, TN, United States, ${ }^{9}$ Biomedical Engineering, Vanderbilt University, Nashville, TN, United States

Chemical exchange saturation transfer (CEST) magnetic resonance imaging (MRI) can probe tissue biochemistry in vivo with high resolution and sensitivity without requiring exogenous contrast agents. Applying CEST MRI at ultrahigh field provides advantages of increasing spectral resolution and improving sensitivity to metabolites with faster proton exchange rates such as glutamate, a critical neurotransmitter in the brain. Prior magnetic resonance spectroscopy and CEST MRI studies have revealed altered regulation of glutamate in patients with multiple sclerosis (MS). While CEST imaging facilitates new strategies for investigating the pathology underlying this complex and heterogeneous neurological disease, CEST signals are contaminated or diluted by concurrent effects (e.g., semi-solid magnetization transfer (MT) and direct water saturation) and are scaled by the $T_{1}$ relaxation time of the free water pool which may also be altered in the context of disease. In this study of 20 relapsing-remitting MS patients and age- and sex-matched healthy volunteers, glutamate-weighted CEST data were acquired at 7.0T. A Lorentzian fitting procedure was used to remove the asymmetric MT contribution from CEST z-spectra, and the apparent exchange-dependent relaxation (AREX) correction was applied using an $\mathrm{R}_{1}$ map derived from an inversion recovery sequence to further isolate glutamate-weighted CEST signals from concurrent effects. Associations between AREX and cognitive function were examined using the Minimal Assessment of Cognitive Function in MS battery. After isolating CEST effects from MT, direct water saturation, and $T_{1}$ effects, glutamate-weighted AREX contrast remained higher in gray matter than in white matter, though the difference between these 
tissues decreased. Glutamate-weighted AREX in normal-appearing gray and white matter in MS patients did not differ from healthy gray and white matter but was significantly elevated in white matter lesions. AREX in some cortical regions and in white matter lesions correlated with disability and measures of cognitive function in MS patients. However, further studies with larger sample sizes are needed to confirm these relationships due to potential confounding effects. The application of MT and AREX corrections in this study demonstrates the importance of isolating CEST signals for more specific characterization of the contribution of metabolic changes to tissue pathology and symptoms in MS.

Keywords: chemical exchange saturation transfer (CEST), glutamate, multiple sclerosis, metabolic imaging, ultrahigh field, apparent exchange-dependent relaxation (AREX), cognition

\section{INTRODUCTION}

Cognitive impairment is a significant symptom of relapsingremitting multiple sclerosis (RRMS), affecting $40-70 \%$ of patients and occurring early in the disease course. Affected domains include information processing speed, memory, verbal fluency and executive function (1). Despite a significant impact on quality of life, the pathological substrates of cognitive impairment are poorly understood. Understanding its underlying mechanisms would help inform therapeutic decisions and advance development of adequate interventions and therapies.

While lesion load and location in cortical and deep gray matter (GM) and in white matter (WM) tracts have all shown associations with cognitive status, specific deficits, and cognitive deterioration over time (2), fully understanding the complex pathophysiology of MS requires imaging methods more specific to metabolic changes. Magnetic resonance imaging (MRI) methods that can probe brain pathophysiology and metabolism in vivo are needed to elucidate the mechanisms that underlie cognitive impairment.

Glutamate is the main excitatory neurotransmitter in the brain; dysregulation of glutamate homeostasis is implicated in various neuropsychiatric disorders. In MS, glutamate excitotoxicity, i.e., axonal damage and neuronal death caused by increased extracellular glutamate levels, has been implicated as a potential mechanism linking inflammation and neurodegeneration $(3,4)$. Previous investigations have used in vivo proton MR spectroscopy (1H-MRS) to probe several

\footnotetext{
Abbreviations: CEST, chemical exchange saturation transfer; AREX, apparent exchange-dependent relaxation; MTRasym, magnetization transfer ratio asymmetry; $\mathrm{MTR}_{\mathrm{Rex}}$, magnetization transfer ratio employing inverse Z-spectrum; MT, magnetization transfer; MP2RAGE, magnetization prepared 2 rapid acquisition gradient echoes; MP-FLAIR, magnetization prepared fluid-attenuated inversion recovery; TFE, Turbo Field Echo; TSE, Turbo Spin Echo; SENSE, sensitivity encoding; GM, gray matter; WM, white matter; MS, multiple sclerosis; EDSS, Expanded Disability Status Scale; WRAT4, Wide Range Achievement Test $4^{\text {th }}$ edition; CVLT-II, California Verbal Learning Test $2^{\text {nd }}$ edition; SDMT, Symbol Digit Modalities Test; BVMT-R, Brief Visuospatial Memory Test-Revised; PASAT, Paced Auditory Serial Addition Test; D-KEFS ST, Delis-Kaplan Executive Function System Sorting Test; COWAT, Controlled Oral Word Association Test.
}

metabolites, including glutamate, in MS lesions and normalappearing white matter (NAWM) (5). Glutamate was found to be elevated in acute WM lesions and NAWM compared to healthy control tissue (6), and an increased glutamate/ $N$-acetylaspartate ratio in NAWM was predictive of longitudinal declines in brain volume, MS Functional Composite scores, and Paced Auditory Serial Addition Test (PASAT) scores (a commonly-used test of processing speed) at 5-year follow-up (7). In contrast, lower glutamate levels measured with MRS in GM regions have been correlated with worse visuospatial memory (8).

Chemical exchange saturation transfer (CEST) MRI provides another means to perform molecular imaging and detect endogenous, mobile biomolecules such as proteins, peptides, and metabolites (e.g., glutamate, creatine, myo-inositol) with high sensitivity. In CEST imaging, the water-exchangeable protons of solutes (e.g., amide, hydroxyl, amine, or imino protons) are labeled via saturation with frequency-selective, narrowbandwidth RF irradiation. Forward and back chemical exchange between protons in the irradiated solute pool and protons in the bulk water pool are constantly occurring. Through repeated saturation and exchange, saturated protons accumulate in the water pool and decrease the water signal, which can be detected (9-11). Physiologically relevant, millimolar concentrations of endogenous molecules can be detected with CEST MRI given sufficient saturation and chemical exchange rate (12). Ultrahigh field MRI (7.0 Tesla and higher) is advantageous for CEST imaging because the spectral resolution and sensitivity to faster proton exchange rates are improved, and a longer water $T_{1}$ increases the CEST effect (10). These features are important to glutamate-weighted CEST ("GluCEST") (13-16) due to the intermediate/fast exchange rate of glutamate amine protons and resonance frequency $(\Delta \omega=3.0 \mathrm{ppm})$ closer to that of water relative to other common CEST targets (e.g., slow-exchanging amide protons in proteins at $\Delta \omega=3.5 \mathrm{ppm}$ ).

CEST MRI has been applied in many preclinical and clinical studies of diseases, including MS (16-21), to probe changes in tissue biochemistry with greater coverage and resolution relative to other metabolic imaging techniques such as MR spectroscopy. Nevertheless, it is important to note that several factors can confound or dilute the CEST contrast attributed to a molecule of interest. CEST is sensitive to the choice of saturation parameters 
(saturation pulse power and duration), field strength, direct water saturation (spillover effect), and $\mathrm{T}_{1}$ relaxation time of the free water pool. The semisolid macromolecular components in tissues participate in magnetization transfer (MT) which imparts a broad, asymmetric baseline convolved on the measured CEST spectrum $(12,22)$. Of note, the MT asymmetry is substantially larger than the CEST effect, which can diminish the detection of small changes in the CEST spectrum. Conventional approaches to quantifying CEST data involve computing the magnetization transfer ratio (MTR) asymmetry which compares the signal at the frequency of interest $(\Delta \omega)$ to that at a reference frequency (typically $-\Delta \omega$ ) after correction for $\mathrm{B}_{0}$ inhomogeneities. This analysis does not account for the broad, asymmetric baseline in the CEST spectrum and does not isolate the CEST signal from other tissue changes that may occur simultaneously in disease [e.g., demyelination, water content changes due to inflammation and edema $(23,24)]$. Several methods to isolate CEST contrast for a target molecule from the confounding spillover, $\mathrm{MT}$, and $\mathrm{T}_{1}$ effects have been developed, including modeling with modified Bloch-McConnell equations (25), fitting the CEST spectrum with multiple Lorentzian line shapes $(26,27)$, and inverse $\mathrm{z}$-spectrum analysis approaches (28). The apparent exchange-dependent relaxation (AREX) correction for CEST quantification is one such correction that has been shown to reduce the influence of these competing effects (28-31).

Our prior study of glutamate-weighted CEST MRI in MS using a conventional asymmetry analysis showed increased GluCEST contrast in the prefrontal cortex of patients with MS relative to controls, and GluCEST in cortical regions correlated negatively with measures of cognitive function (16). In the current study, we sought to examine glutamate-weighted CEST at 7.0 T in patients with MS with two additional measures taken to isolate the CEST signal from confounding effects. First, we used a Lorentzian fitting procedure to remove the asymmetric MT contribution from CEST z-spectra. We then applied the AREX correction to reduce the influences of spillover, $\mathrm{MT}$, and $\mathrm{T}_{1}$ effects on CEST signal quantification. MT-corrected AREX contrast in NAWM and normal-appearing GM (NAGM) and in MS lesions was compared to healthy tissue, and associations with clinical measures of disability and cognitive impairment were examined.

\section{MATERIALS AND METHODS Study Participants}

The studies involving human participants were reviewed and approved by the Vanderbilt University Institutional Review Board, and the participants provided their written informed consent prior to examination. Twenty patients with a diagnosis of relapsing-remitting MS according to the revised 2010 McDonald Criteria (32) (19-56 years old, mean age $38.5 \pm 10.6$ years, 12F/8M, Expanded Disability Status Scale (EDSS) score range of $0-4.5$ and median of 1.5 determined by clinical examination) and 20 age- and sex-matched healthy volunteers (23-57 years old, mean age $39.2 \pm 10.8$ years, $13 \mathrm{~F} / 7 \mathrm{M})$ were enrolled. All participants underwent a brain MRI at 7 Tesla $(7.0 \mathrm{~T})$ field strength, and a subset of the participants completed a neuropsychological assessment using the Minimal Assessment
TABLE 1 | Demographic information.

\begin{tabular}{lcc}
\hline & $\begin{array}{c}\text { MS patients } \\
(\boldsymbol{n}=\mathbf{2 0})\end{array}$ & $\begin{array}{c}\text { Healthy volunteers } \\
(\boldsymbol{n}=\mathbf{2 0})\end{array}$ \\
\hline Females & $12(60 \%)$ & $13(65 \%)$ \\
Age, mean \pm SD & $38.5 \pm 10.6$ & $39.2 \pm 10.8$ years \\
& years & \\
Years of education, mean \pm SD & $15.4 \pm 2.9$ & $17.2 \pm 2.3$ years \\
& years* & $112.1 \pm 9.3$ \\
WRAT4 word reading & $108.5 \pm 7.5$ & \\
age-corrected standard score, & & \\
mean \pm SD & & \\
EDSS, median (range) & $1.5(0-4.5)$ & - \\
Disease duration, mean \pm SD & $8.8 \pm 7.9$ & \\
(range) & years $(0.5-29)$ & \\
\end{tabular}

MS, multiple sclerosis; EDSS, Expanded Disability Status Scale.

WRAT4 the Wide Range Achievement Test, $4^{\text {th }}$ edition, used as an estimate of premorbid ability.

*Patients with MS differ significantly from healthy volunteers $(p<0.05)$.

of Cognitive Function in MS (MACFIMS) battery (33) and two additional tests of processing speed and motor speed [Simple Reaction Time with one stimulus and Choice Reaction Time with four stimuli (34)]. Only the 2 second interval version of the PASAT was administered. Nineteen healthy volunteers and $15 \mathrm{MS}$ patients completed the neuropsychological battery, and the remaining 5 patients completed a subset of tests. Complete demographic data are shown in Table 1. The Word Reading score from the Wide Range Achievement Test, $4^{\text {th }}$ edition (WRAT4), was used as an estimate of premorbid ability and age-corrected, standard scores did not differ between groups.

\section{MR Imaging}

Brain MRI was performed using a Philips Achieva 7.0T MR Scanner (Philips Healthcare, The Netherlands) with a twochannel volume transmit, 32-channel receive head coil (Nova Medical, Wilmington, MA). The scan protocol included two whole brain anatomical scans for tissue segmentation. A threedimensional (3D) magnetization prepared 2 rapid acquisition gradient echoes (MP2RAGE) (35) was acquired with a 3D Turbo Field Echo (TFE) sequence, $0.8 \mathrm{~mm}$ isotropic resolution, $\mathrm{MP} 2 \mathrm{RAGE}$ TR $=8.25 \mathrm{~s}, \mathrm{TR}=6.0 \mathrm{~ms}, \mathrm{TI}_{1}=1 \mathrm{~s}, \mathrm{TI}_{2}=3.3 \mathrm{~s}, \mathrm{TE}$ $=2.6 \mathrm{~ms}$, SENSE factor $=2 \mathrm{AP}, 2 \mathrm{RL}, \mathrm{FA}_{1}=\mathrm{FA}_{2}=4^{\circ}$, and 9 min:04 s duration. A 3D magnetization prepared fluid-attenuated inversion recovery (MP-FLAIR) (36) was acquired with a 3D Turbo Spin Echo (TSE) sequence, $0.8 \mathrm{~mm}$ isotropic resolution, $\mathrm{TR}=8 \mathrm{~s}$, TI $=2425 \mathrm{~ms}, \mathrm{TE}=278 \mathrm{~ms}$, SENSE factor $=2 \mathrm{AP}, 2$ $\mathrm{RL}, \mathrm{FA}=70^{\circ}$, and $9 \mathrm{~min}: 36 \mathrm{~s}$ duration.

For glutamate-weighted CEST MRI, a 2D multi-shot TFE sequence was applied in a transverse orientation parallel to the anterior commissure - posterior commissure line with $1.5 \times 1.5$ $\mathrm{x} 10 \mathrm{~mm}^{3}$ resolution, $\mathrm{TR}=4.1 \mathrm{~ms}, \mathrm{TE}=2.7 \mathrm{~ms}$, SENSE factor $=2(\mathrm{RL}), \mathrm{FA}=10^{\circ}$, number of signal averages $=2$, and 22 min:12 s duration. CEST data were acquired using a $4.25 \mu \mathrm{T}$ (peak amplitude) pulse train of ten $60 \mathrm{~ms}$ Gaussian RF pulses (90\% duty cycle, $B_{1 \mathrm{rms}}=1.97 \mu \mathrm{T}$ over $670 \mathrm{~ms}$ pulse train) at 
43 frequency offsets sampled asymmetrically between $+/-5.0$ ppm $(-5.0,-4.6,-4.3,-4.0,-3.6,-3.3,-3.0,-2.6,-2.3$, $-2.0,-1.6,-1.3,-1.0,-0.8,-0.6,-0.4,-0.2,0.0,0.2,0.4$, $0.6,0.8,1.0,1.2,1.4,1.6,1.8,2.0,2.2,2.4,2.6,2.8,3.0,3.2,3.4$, $3.6,3.8,4.0,4.2,4.4,4.6,4.8$, and $5.0 \mathrm{ppm}$ ) with 13 interspersed, non-saturated reference images $\left(\mathrm{S}_{0}\right)$ to correct for signal drift. A $B_{1}$ map (dual-TR actual flip angle method, $\mathrm{TR}_{1} / \mathrm{TR}_{2} / \mathrm{TE} / \mathrm{FA}$ $\left.=35 \mathrm{~ms} / 160 \mathrm{~ms} / 2.0 \mathrm{~ms} / 60^{\circ}\right)$ (37) and a Water Saturation Shift Referencing (WASSR) sequence (38) were acquired for correction of $B_{1}$ and $B_{0}$ field inhomogeneities, respectively. For the WASSR sequence, a $0.5 \mu \mathrm{T}, 100 \mathrm{~ms}$ Gaussian-shaped RF saturation pulse was applied at frequency offsets between $+/-1.5$ ppm with a step size of $0.1 \mathrm{ppm}$ from $+/-1.5$ to $+/-1.0 \mathrm{ppm}$ and $0.05 \mathrm{ppm}$ between $+/-1.0 \mathrm{ppm}$ with the following parameters: $2 \times 2 \times 10 \mathrm{~mm}^{3}$ resolution, $\mathrm{TR}=5.6 \mathrm{~ms}, \mathrm{TE}=2.7 \mathrm{~ms}, \mathrm{FA}=$ $10^{\circ}$, number of signal averages $=1$, and 2 min:54 s duration. An $\mathrm{R}_{1}$ map for relaxation rate correction of CEST data was derived from an inversion recovery sequence acquired with the following parameters: a selective inversion recovery with a $3 \mathrm{D}$ multi-shot TFE readout, 5 slices, $1.1 \times 1.1 \times 2 \mathrm{~mm}^{3}$ resolution, $\mathrm{TR}=4.1 \mathrm{~ms}$, $\mathrm{TE}=2.1 \mathrm{~ms}$, SENSE factor $=2(\mathrm{RL}), \mathrm{FA}=15^{\circ}, 14 \mathrm{TI}$ values $(6,10,16,26,42,68,110,178,288,468,760,1233,2000$, and $8000 \mathrm{~ms}$ ), pre-delay time of $2500 \mathrm{~ms}$, composite inversion pulse duration $=6.5 \mathrm{~ms}$, and $7 \mathrm{~min}$ total duration (39-41). The $\mathrm{B}_{1}$, WASSR, and inversion recovery sequences were acquired in the same geometry as the CEST slice.

\section{CEST Image Processing and Effect Isolation}

Each CEST dynamic was registered to the first CEST dynamic using affine registration [FSL FLIRT $(42,43)]$ and normalized to a spline interpolation of the unsaturated $S_{0}$ data to correct for signal drift (44) and generate the CEST z-spectrum $(\boldsymbol{Z})$ for each voxel:

$$
Z(\Delta \omega)=\frac{S(\Delta \omega)}{S_{0}(\Delta \omega)}
$$

$A B_{0}$ frequency shift map was computed from the WASSR data and used to center the CEST $\mathrm{z}$-spectra at $\Delta \omega=0 \mathrm{ppm}$ on a voxel-wise basis (38). In prior studies, glutamate-weighted CEST (GluCEST) contrast was computed according to the following equation:

$$
\text { GluCEST }=\frac{S(-\Delta \omega)-S(+\Delta \omega)}{S(-\Delta \omega)} \times 100
$$

where $\Delta \omega$ is $3.0 \mathrm{ppm}$ for sensitivity to glutamate amine protons $(13,16,45)$. However, this CEST asymmetry equation does not completely remove the asymmetric MT effects from semi-solid macromolecules (e.g., myelin) which are known to be altered in MS pathology $(23,46-48)$. The AREX approach to quantifying CEST contrast mitigates some of the contributions of semi-solid MT effects, direct water saturation, and $\mathrm{T}_{1}$ weighting through an inverse subtraction analysis and correction for $\mathrm{T}_{1}$ effects (28-31), and recent studies aiming to improve the specificity of GluCEST have also incorporated a Lorentzian fitting procedure to remove the asymmetric MT baseline from the CEST spectra
TABLE 2 | Initial estimates and bounds for Lorentzian fit of CEST data.

\begin{tabular}{lccccc}
\hline & \multicolumn{2}{c}{ Amplitudes (\%) } & & \multicolumn{2}{c}{ Width (ppm) } \\
\cline { 2 - 3 } \cline { 5 - 6 } & DS & MT & & DS & MT \\
\hline Lower bound & 20 & 0 & & 0.1 & 10 \\
Upper bound & 100 & 90 & & 5 & 100 \\
Initial guess & 60 & 35 & & 2.55 & 55 \\
\hline
\end{tabular}

(14, 45). Here, we combined MT baseline removal and AREX quantification to probe the CEST effects at $3.0 \mathrm{ppm}$ using the following procedure. First, a two-pool Lorentzian model consisting of MT and direct saturation (DS) pools was fit to the $\mathrm{B}_{0}$-corrected $\mathrm{z}$-spectra on a voxel-wise basis. The labeled $\mathrm{Z}$-spectrum saturation $\left(\boldsymbol{Z}_{\boldsymbol{l a b}}\right)$ can be represented as a baseline saturation $\left(Z_{\text {base }}\right.$, which would be 1 with perfect saturation efficiency) minus the Lorentzian components $\left(\boldsymbol{L}_{\boldsymbol{i}}\right)$ :

$$
Z_{\text {lab }}=Z_{\text {base }}-\sum_{i=1}^{n} L_{i}(\Delta \omega)
$$

where the Lorentzian line shape is represented by (49):

$$
L_{i}(\Delta \omega)=100-\left(\frac{A_{n}}{1+4\left(\frac{\Delta \omega-\Delta \omega_{n}}{\sigma_{n}}\right)^{2}}\right)
$$

For each Lorentzian line shape included in the model, there are three unknown parameters: amplitude $\left(\boldsymbol{A}_{n}\right)$, width $\left(\sigma_{n}\right)$, and chemical shift $\left(\Delta \omega_{n}\right)$ of the $n$th pool, and $\Delta \omega$ is the frequency of the off-resonance pulse. We opted to set fixed values for the chemical shift of each species, as these resonance frequencies are well established by previous research $(50,51)$. Chemical shifts of each species were set as: DS $=0 \mathrm{ppm}$ and $\mathrm{MT}=-2.4$ ppm. Table 2 contains the starting points and boundaries of the fit of the amplitude and width parameters. Initial values for simulations were based on Singh et al. (52). Z-spectral fitting was performed using the non-linear fitting "lsqnonlin" built-in MATLAB function. Once the fitting algorithm was complete, the MT contribution was removed from the measured CEST spectrum $(\boldsymbol{Z})$ by subtracting the MT spectrum (the Lorentzian line shape from Equation 4 for the MT pool, which we have termed $Z_{\text {LorentzMT }}$ ) for each voxel, creating the corrected, normalized z-spectrum $\left(Z_{\text {corr }}\right)$ :

$$
Z_{\text {corr }}(\Delta \omega)=Z(\Delta \omega)-Z_{\text {LorentzMT }}(\Delta \omega)
$$

From the MT-corrected z-spectra, we calculated the corrected MTR asymmetry conventionally used to quantify CEST effects (30):

$$
\text { MTRasym }_{\text {corr }}=Z_{\text {corr }}(-\Delta \omega)-Z_{\text {corr }}(+\Delta \omega)
$$

To remove $\mathrm{MT}$, DS, and $\mathrm{T}_{1}$ effects and provide a more exchange-specific quantification of the CEST signal, we 
performed an inverse subtraction analysis and calculated the MT-corrected inverse $\mathrm{z}$-spectrum (Equation 7), MTand spillover-corrected inverse difference (Equation 8, $\boldsymbol{M T R}_{\text {Rex Corr }}$ ), and $\mathrm{T}_{1}$-corrected AREX contrast (Equation

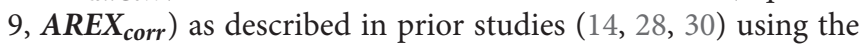
following equations:

$$
\begin{aligned}
\frac{1}{Z_{\text {corr }}} & =\frac{1}{Z(\Delta \omega)}-\frac{1}{Z_{\text {LorentzMT }}(\Delta \omega)} \\
\text { MTR }_{\text {Rex Corr }} & =\frac{1}{Z_{\text {corr }}(+\Delta \omega)}-\frac{1}{Z_{\text {corr }}(-\Delta \omega)} \\
\text { AREX }_{\text {corr }} & =\left(\frac{1}{Z_{\text {corr }}(+\Delta \omega)}-\frac{1}{Z_{\text {corr }}(-\Delta \omega)}\right) / T_{1} \\
& =M T R_{\text {RexCorr }} \times R_{1}
\end{aligned}
$$

where $\Delta \omega$ is $3.0 \mathrm{ppm}$ for sensitivity to glutamate amine protons as before $(13,14,16,45)$. The $\mathrm{R}_{1}$ (longitudinal relaxation rate of the free water pool) values used in the AREX equation were derived from fitting the inversion recovery data as described previously (39-41). The five $2 \mathrm{~mm}$-thick $\mathrm{R}_{1}$ map slices were averaged and registered to the $10 \mathrm{~mm}$-thick CEST slice with affine registration using the $16^{\text {th }}$ CEST dynamic at $\Delta \omega=-0.4 \mathrm{ppm}$ as the target image. Registration results were checked in all cases using the target image (CEST dynamic at $-0.4 \mathrm{ppm}$ ) as well as images at other offset frequencies (e.g., $-3.0 \mathrm{ppm}$ ).

\section{Tissue Segmentation}

$\mathrm{T}_{1}$-weighted MP2RAGE images were calculated from the complex image volumes acquired at $\mathrm{TI}_{1}$ and $\mathrm{TI}_{2}$ using the robust processing method described by O'Brien et al. (53). GM and WM tissue maps were segmented from the 3D MP2RAGE image using the "segment" tool in SPM12. Default settings were modified to improve performance on the 7.0 T MP2RAGE images which are inherently corrected for field inhomogeneities (bias regularization = "no regularization," bias FWHM = "no correction," and clean up procedure = "thorough"). Multiatlas labeling using the spatially localized atlas network tiles (SLANT) method $(54,55)$ was applied to the MP2RAGE image to further divide the GM into distinct cortical regions (prefrontal, parietal, motor, somatosensory, temporal, and occipital cortices). MS lesions in the cortical GM were segmented manually from MP2RAGE images, and WM lesions were segmented manually using both MP2RAGE and MP-FLAIR images. The anatomical image volumes and associated tissue and lesion masks were registered to the inversion recovery image volume $(\mathrm{TI}=1233 \mathrm{~ms}$ image as target) acquired in the same geometry as the CEST slice using the "Coregister: Estimate and Re-slice" tool with the "Normalized Mutual Information" function and nearestneighbor interpolation in SPM12. Prior to registering these tissue and lesion masks (5 $2 \mathrm{~mm}$-thick slices) to the CEST slice (1 $10 \mathrm{~mm}$-thick slice), masks representing the $10 \mathrm{~mm}$ thick slice were generated as follows: the GM, WM, and WM lesion masks retained voxels that occurred in at least 2 of the 5 slices, and the cortical lesion mask retained all lesion voxels occurring in any of the 5 slices due to the small size of these lesions. Lesion mask voxels were excluded from normal-appearing GM and WM masks. These 2D masks were then registered to the $2 \mathrm{D}$ CEST slice using the same affine transformation that registered the calculated $2 \mathrm{D} \mathrm{R}_{1}$ map to the CEST slice (as described in Section CEST Image Processing and Effect Isolation above).

\section{CEST Analysis}

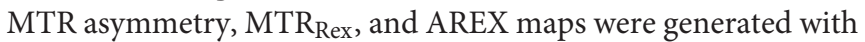
and without the subtraction of the MT baseline component to visualize the influences of the asymmetric MT effect, direct saturation, and $\mathrm{T}_{1}$ on conventional CEST quantification procedures. Histograms of these contrasts calculated at $\Delta \omega=3.0$ ppm were generated for each tissue region of interest (GM, WM, cortical lesions, and WM lesions), and mean and median values were computed for $\mathrm{R}_{1}, \mathrm{MTR}_{\text {asym }}$ corr, $\mathrm{MTR}_{\mathrm{RexCorr}}$, and $\mathrm{AREX}_{\text {corr }}$ in these regions of interest for each participant.

\section{Statistical Analysis}

A two-sample t-test was performed to test differences in demographic variables, cognitive test scores, and CEST contrast indices (for each tissue region of interest) between healthy volunteers and MS patients. Within each group, a paired $t$ test was performed to determine whether CEST indices differed between GM and WM. CEST contrast in white matter lesions and cortical lesions was also compared to the corresponding normal-appearing tissue in MS patients (paired $t$-test) and to $\mathrm{WM}$ and GM in healthy volunteers (two-sample $t$-test). In patients with MS, associations between MT-corrected AREX contrast, $\mathrm{R}_{1}$, and clinical measures of disease status and cognitive function were investigated using a Spearman partial correlation with age, sex, and years of education included as covariates since these variables can influence performance on several cognitive tests. Additionally, some studies have shown age and sex differences in glutamate levels in the brain (56-58). Associations between AREX contrast and cognitive test scores were not examined in the healthy group because variance within physiologic levels of glutamate is expected to be highly regulated and unrelated to cognition in otherwise healthy individuals. Given the exploratory nature of this study to examine glutamateweighted AREX contrast in MS, no correction for multiple comparisons was employed and $t$-test results are reported with cutoff levels of $0.05,0.01$, and 0.001 for comparisons between tissue regions.

\section{RESULTS}

\section{Isolating CEST Effects}

Glutamate-weighted CEST and anatomical images were acquired in all participants without adverse events. In one MS patient we did not complete the inversion recovery sequence, resulting in a missing $\mathrm{R}_{1}$ map for the AREX correction. CEST data from a different MS patient and one healthy volunteer were excluded from analyses due to motion artifact-related noise. The resulting data set for CEST analyses with the AREX quantification method consisted of 19 healthy volunteers and 18 MS patients. Representative anatomical images, CEST images (normalized individual dynamic at $\Delta \omega=-5.0 \mathrm{ppm}$ ), tissue segmentation, and $\mathrm{R}_{1}$ maps are shown for a healthy volunteer and 
a patient with MS in Figure 1. Anatomical images, segmentation masks, and $\mathrm{R}_{1}$ maps were all registered to the $10 \mathrm{~mm}$-thick CEST slice.

To isolate CEST effects from the confounding effects of MT, direct saturation, and $\mathrm{T}_{1}$ changes, we first obtained the MT baseline for each voxel using a 2-pool Lorentzian fit (MT and DS pools), then subtracted the MT spectrum from the measured CEST z-spectrum. Examples of the MT baseline in different tissues as well as the resulting impact on the average $\mathrm{z}$-spectra and inverse z-spectra for GM, WM, and lesions are shown in Figure 2. WM voxels show the greatest influence from asymmetric MT effects on the CEST z-spectra, while GM has a smaller MT influence and CSF has little to no MT contribution, as expected (Figures 2A,B). After subtracting the MT baseline, the tissue differences and broad asymmetry in the $\mathrm{z}$-spectra and inverse $\mathrm{Z}$-spectra are reduced (Figures $2 \mathrm{C}-\mathbf{F}$ ).

MTRasym, MTR Rex, and AREX maps were calculated using Equations 6, 8, 9 with $\Delta \omega=3.0 \mathrm{ppm}$ to target glutamate. Representative maps with and without the MT baseline subtraction are shown in Figure 3. As expected, the CEST indices show larger contrast between GM and WM due to the greater myelin content in WM when the MT influence on the measured CEST spectra is not removed. Additionally, the asymmetric MT baseline with a peak around $\Delta \omega=-2.4$ ppm results in more negative values in the observed CEST asymmetry. After removal of the MT baseline, the difference between GM and WM is visibly reduced but not eliminated, and the values are shifted toward positive values. Quantification of AREX contrast without and with MT baseline removal shows the same effects with data from a representative patient shown in Figure 4. The mean AREX contrast in GM and WM shifts toward positive values and the difference between the tissues is reduced after removal of the MT influence. In this patient, cortical lesions do not show a difference in AREX contrast relative to NAGM after isolating the CEST effects from $\mathrm{MT}$, direction saturation, and $\mathrm{T}_{1}$ contributions, but there is a slight increase in AREX contrast in WM lesions relative to NAWM.

\section{Group Differences in CEST Indices}

Mean and median values for $\mathrm{R}_{1}$ and MT-corrected CEST indices in WM, GM, white matter lesions, and cortical lesions are shown in Figure 5 and Supplementary Figure 1, respectively. Within the healthy volunteer and MS patient groups, MTRasym $\mathrm{MTR}_{\mathrm{RexCorr}}$, and $\mathrm{AREX}_{\mathrm{Corr}}$ values at $\Delta \omega=3.0 \mathrm{ppm}$ were significantly greater in GM than in WM $(p<0.001)$. CEST indices in NAGM and NAWM in MS patients did not differ significantly from healthy GM and WM in the control group, but all CEST indices were significantly elevated in WM lesions relative to NAWM and healthy WM $(p<0.001)$. $\mathrm{R}_{1}$ differed significantly between WM and GM within each group, and $\mathrm{R}_{1}$ was significantly decreased in WM lesions relative to NAWM and healthy WM $(p<0.001)$. NAGM and cortical lesions in MS patients also had significant reductions in $R_{1}$. When the cortex was subdivided into regions, there were no significant differences in $\mathrm{AREX}_{\mathrm{Corr}}$ between MS patients and healthy volunteers. The distributions of $\mathrm{AREX}_{\mathrm{Corr}}$ values in each group and tissue region of interest (ROI) are presented with the average histogram and standard deviation for each group/tissue in Supplementary Figure 2. The distributions were similar between healthy volunteers and MS patients for NAGM and NAWM, while lesion histograms show greater inter-subject variability. $\mathrm{AREX}_{\text {Corr }}$ in WM lesions clearly shifted toward higher values.

\section{Correlations Between MRI Indices, Disability, and Cognitive Function}

Group differences in measures of cognitive function are reported in Table 3. Significant differences were observed for the California Verbal Learning Test $2^{\text {nd }}$ edition total free recall $(p<0.001)$ and long delay free recall $(p<0.01)$, Symbol Digit Modalities Test $(p<0.001)$, Brief Visuospatial Memory Test-Revised delayed recall ( $p<0.05$ ), Controlled Oral Word Association Test $(p<0.001)$, and Simple Reaction Time $(p<$ 0.05). Associations between $\mathrm{R}_{1}$ and $\mathrm{AREX}_{\text {Corr }}$ MRI indices and EDSS score, disease duration, and cognitive function in patients with MS were examined using a Spearman partial correlation analysis with age, sex, and education as covariates. The resulting Spearman rho values are shown in Figure 6, with significant correlations highlighted in yellow $(p<0.05)$. Mean $\mathrm{R}_{1}$ values in MS NAWM correlated positively with 2-s PASAT scores (rho = 0.62 ), and $R_{1}$ values in cortical lesions correlated negatively with Choice Reaction Time (rho $=-0.68$ ). EDSS scores correlated positively with mean $\mathrm{AREX}_{\text {Corr }}$ values in the occipital cortex (rho $=0.60)$. Significant negative correlations were detected between visuospatial memory (BVMT-R) scores and mean AREX Corr values in the motor and somatosensory cortices (rho values of $-0.63,-0.54$, and -0.58 ). Finally, mean AREX Corr $_{\text {in }} \mathrm{WM}$ lesions correlated positively with scores on the D-KEFS Sorting Test $($ rho $=0.84)$. AREX $_{\text {Corr }}$ and $\mathrm{R}_{1}$ values within each tissue ROI were not correlated with each other (Supplementary Figure 3).

\section{DISCUSSION}

In this study, glutamate-weighted CEST MRI was applied at 7.0 T field strength with corrections for the confounding influences of MT, spillover, and $\mathrm{T}_{1}$ effects. We first demonstrated the effect of removing the asymmetric MT baseline from CEST z-spectra, then sought to assess whether glutamate-sensitive, MT-corrected AREX contrast is sensitive to pathology in the brain in MS by (1) comparing AREX in MS lesions, NAGM, and NAWM to healthy GM and WM, and (2) exploring correlations between AREX and measures of disease status and cognitive impairment.

\section{Effects of MT and AREX Corrections on Glutamate-Weighted CEST}

We found that fitting the CEST z-spectrum with a Lorentzian line shape removes the broad asymmetry that is attributed to MT from the semisolid macromolecular components within tissue (e.g., myelin) (Figure 2). Prior studies of GluCEST in the brain have relied on an asymmetry analysis for quantification of CEST contrast (Equation 2) $(13,15,16,59,60)$, which does not completely isolate the CEST effects of metabolites from competing effects, especially when high irradiation powers are 

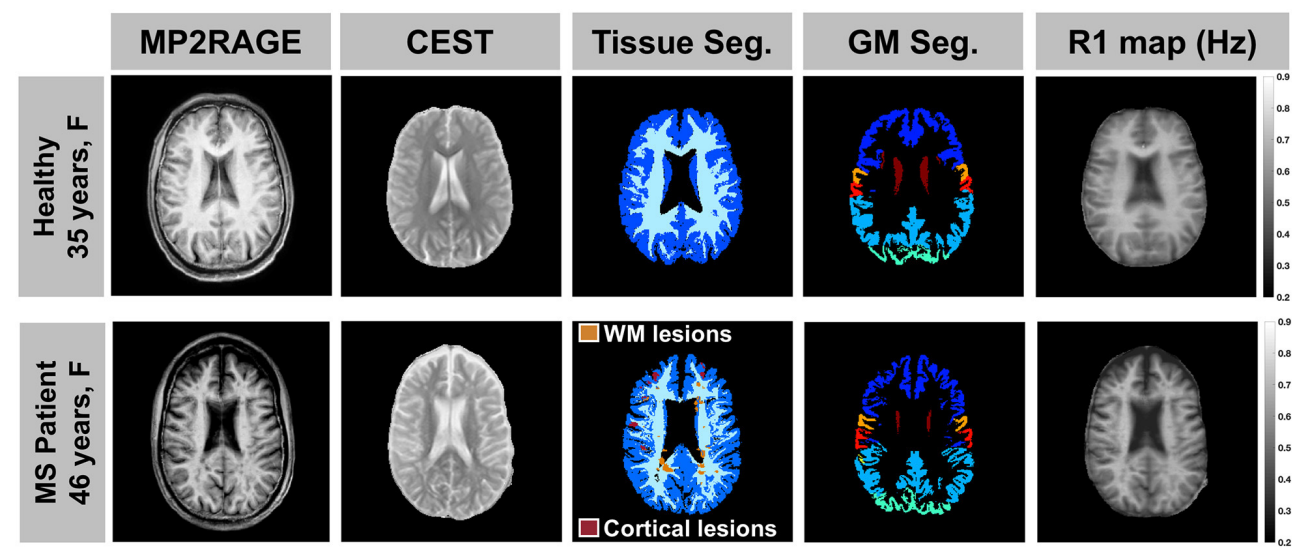

FIGURE 1 | A representative anatomical MP2RAGE image, CEST image (individual dynamic at $\Delta \omega=-5.0$ ppm normalized to the unsaturated $\mathrm{S}_{0}$ data), tissue segmentation, and $\mathrm{R}_{1}$ map are shown for a healthy volunteer (top: 35-year-old female) and a patient with MS (bottom: 46-year-old female, EDSS = 2, duration = 1 year). All image volumes were registered to the $10 \mathrm{~mm}$-thick CEST slice.

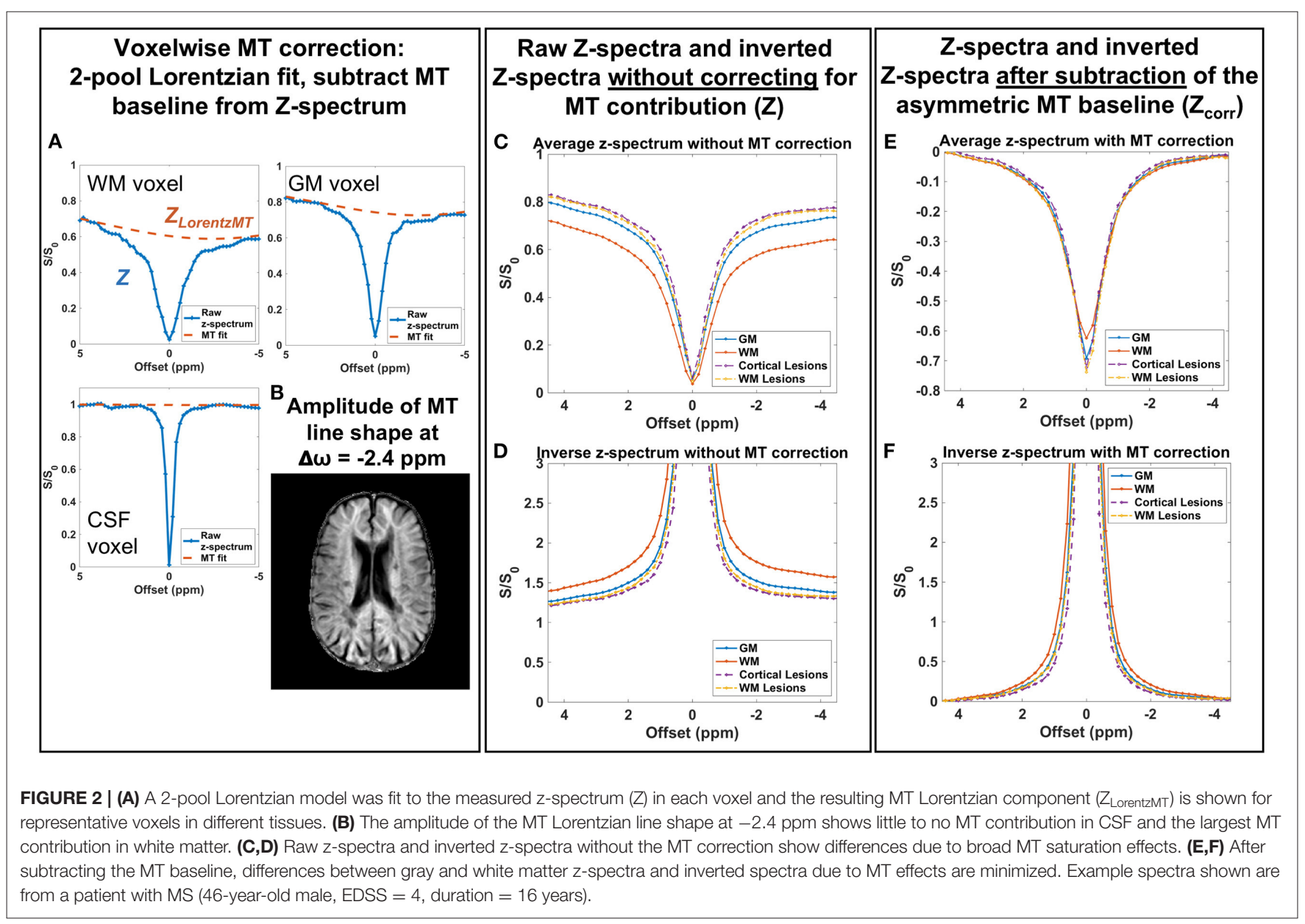

applied. Because MS is a demyelinating disease, it is important to address the influence of myelin changes (46-48) and its impact on the CEST spectrum to more selectively study metabolic changes contributing to disease pathology. Lorentzian fitting approaches for removing the MT component were recently demonstrated for GluCEST in the healthy human brain at 7.0 T (45), in the healthy rat brain at 9.4T (14), and in the rat brain with a tumor at $9.4 \mathrm{~T}(45)$. As in the current work, these prior 

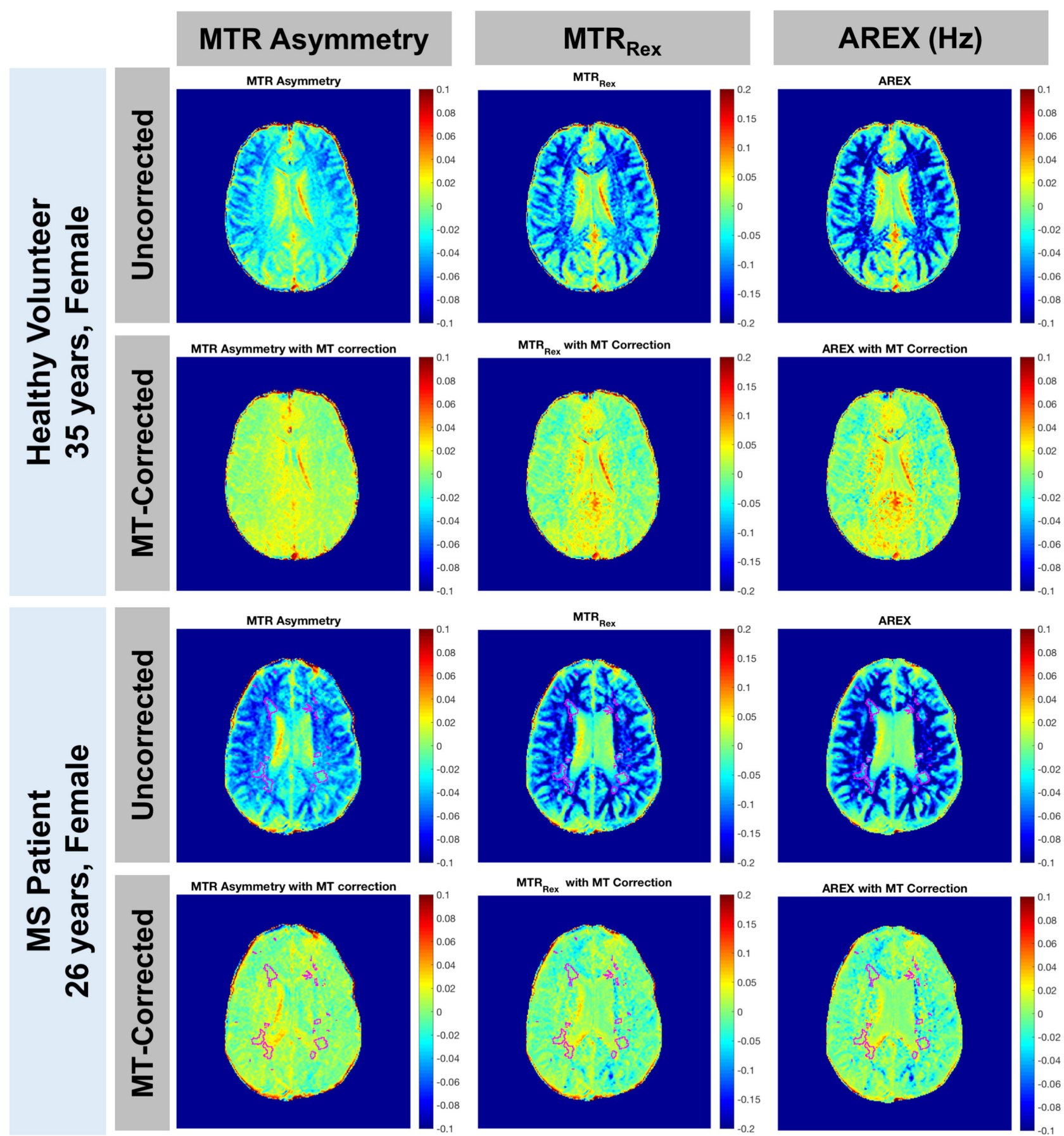

FIGURE 3 | MTR asymmetry, MTRRex, and AREX maps for $\Delta \omega=3.0 \mathrm{ppm}$ are shown with and without the MT baseline removal for a representative healthy volunteer (rows 1 and 2: 35-year-old female) and a patient with MS (rows 3 and 4: 26-year-old female, EDSS $=0$, duration = 6 years). Before removing the MT contribution, there is a greater difference between GM and WM for all 3 CEST indices (rows 1 and 3). After removal of the asymmetric effect, CEST contrast shifts toward positive values and the difference between GM and WM is reduced but still present (rows 2 and 4). Lesions are outlined in the patient images.

studies found that removal of the MT baseline resulted in a decrease in the difference in CEST signal between GM and WM. Our results show that although the quantitative CEST indices (MTRasym, MTR $_{\text {Rex }}$, and AREX) have less GM:WM contrast after MT correction, the average CEST signal at $3.0 \mathrm{ppm}$ in GM is still significantly greater than that in WM (Figures 4, 5). This result is in agreement with MT-corrected GluCEST applied in healthy volunteers at $7.0 \mathrm{~T}$ by Debnath et al. (45) and is 


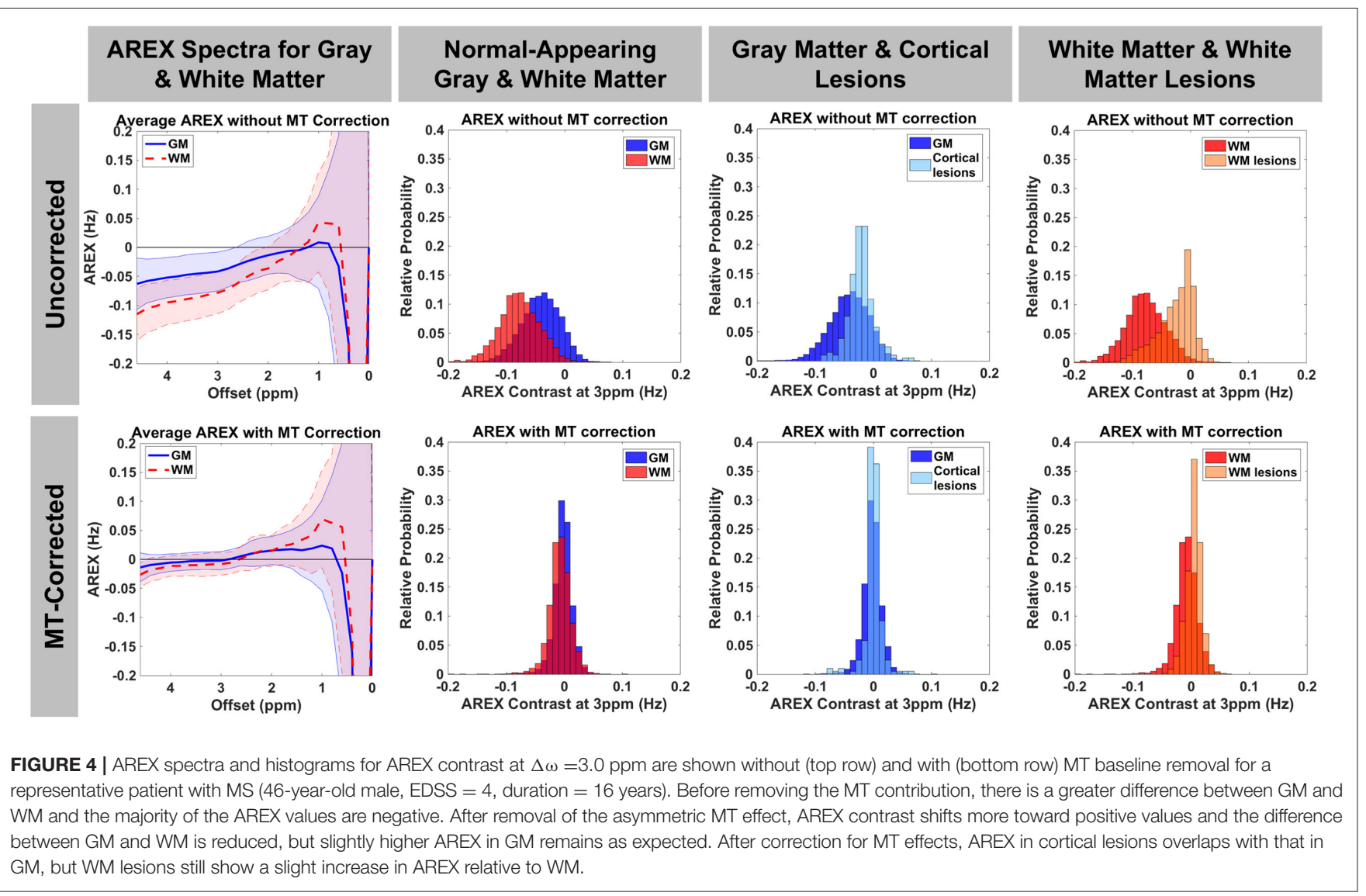

presumed to be due to the higher concentration of glutamate in GM. Cui et al. also found that MTRasym at $3.0 \mathrm{ppm}$ remained significantly greater in GM than in WM in the rat brain after the MT correction, but this tissue difference did not persist for AREX quantification and there was no difference in water $T_{1}$ between GM and WM (14). In our study, we observed differences in $\mathrm{R}_{1}\left(1 / \mathrm{T}_{1}\right)$ between $\mathrm{GM}$, WM, and lesions (Figure 5) which correspond with prior literature and support the need for the AREX correction to produce CEST contrast more reflective of the metabolite of interest (30). Cui et al. also performed phantom experiments to confirm that the CEST signal from glutamate at $3.0 \mathrm{ppm}$ dominates that from other brain metabolites, and they used rat brain tissue homogenate dialysis experiments to show that amine protons on protein lysine residues also contribute significantly to CEST signal at $3.0 \mathrm{ppm}$ (14). Potential causes for the variability between our MT-corrected CEST results and these prior studies include differences in cohort (human or rat), field strength, CEST saturation parameters, and CEST quantification method applied after the MT baseline removal [conventional GluCEST equation (45) or AREX (14)].

In this work, we used the MT-corrected signal at the opposite frequency $(-3.0 \mathrm{ppm})$ as the reference for computing MTRasym and AREX contrast at the glutamate resonance frequency of 3.0 ppm. Without MT correction, these signals are contaminated by MT effects which are broad and peak near $-2.4 \mathrm{ppm}$. After removal of the MT baseline, it is still possible that our reference signal could be confounded by relayed nuclear Overhauser enhancement (rNOE) effects which are centered around -3.5 ppm but range from -2 to $-5 \mathrm{ppm}(28,44,45)$. Some prior AREX studies have employed a 5-pool multi-Lorentzian fitting approach to quantify the amplitude of a given CEST peak $(30,61)$. This approach is especially useful for amide proton transfer (APT)-weighted CEST since that frequency of interest is 3.5 ppm and use of the signal at $-3.5 \mathrm{ppm}$ as a reference would be confounded by rNOE effects. This approach requires estimating peak frequencies, amplitudes, and widths for each pool and has been shown to work well for CEST data with high spectral resolution. In the current study, the signal at $-3.0 \mathrm{ppm}$ was chosen as the reference for quantifying glutamate-weighted CEST contrast since high saturation powers (e.g., $2.9 \mu \mathrm{T}$ and higher) have been shown to attenuate the rNOE contributions to the $\mathrm{z}$ spectra in both GM and WM $(45,62)$, and our nominal saturation power was $4.25 \mu \mathrm{T}$. While greater saturation pulse power is necessary for targeting protons with faster exchange rates such as the amine protons on glutamate, future studies could examine the tradeoffs between the increase in spectral resolution and the decrease in sensitivity to glutamate that would occur when decreasing the saturation pulse $B_{1}$ amplitude.

\section{CEST Signal Differs in WM Lesions Independently of Changes in MT and $\mathbf{R}_{\mathbf{1}}$}

Changes in glutamate homeostasis have been linked to the pathophysiology of disease progression in MS by prior studies in animal models $(21,63-65)$ and MRS studies in MS patients 

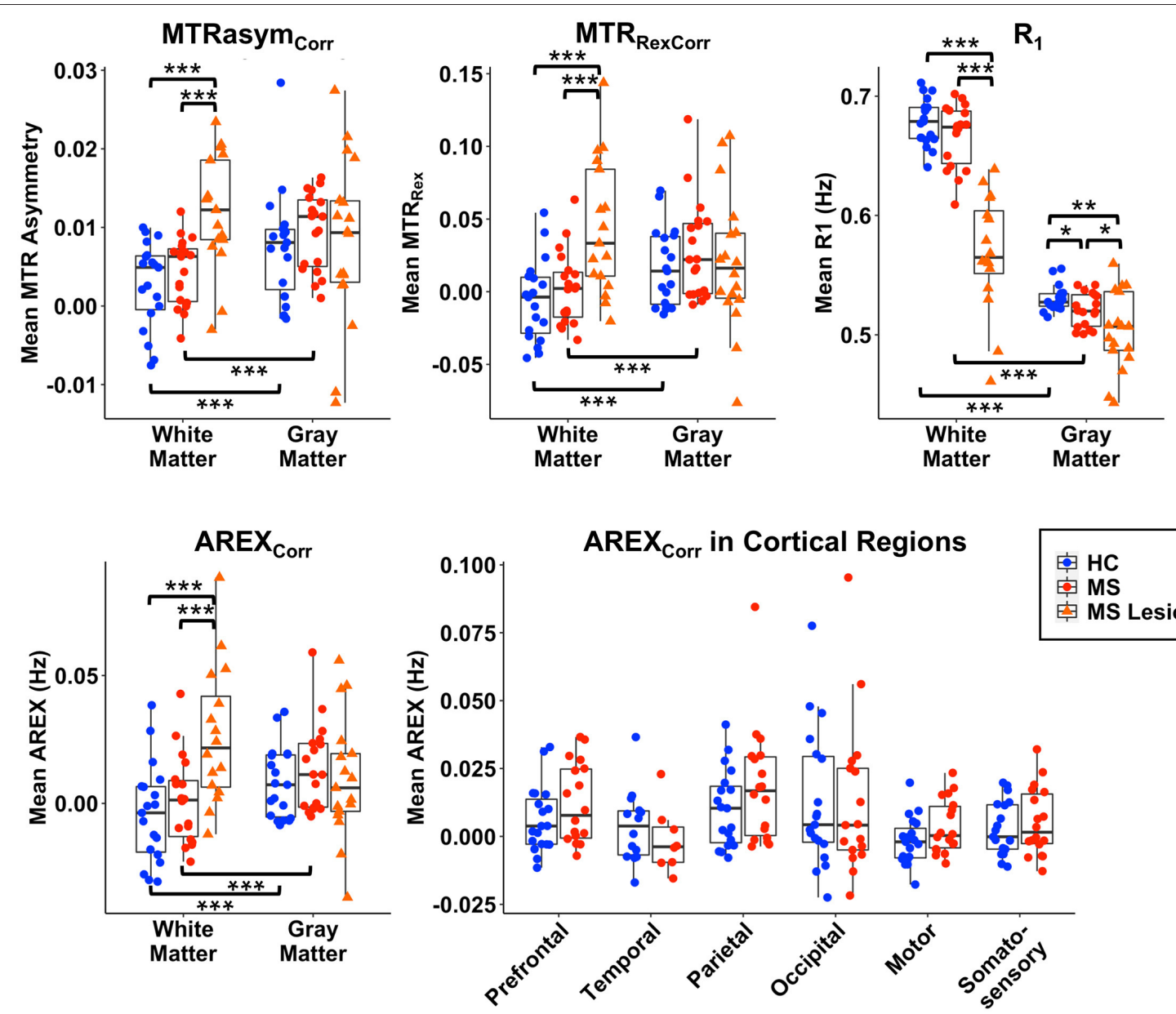

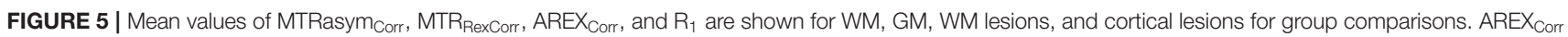
values are also shown for each cortical GM region. WM lesion values differ significantly from normal-appearing WM in MS patients and from healthy control (HC) WM for all CEST-derived indices and for $\mathrm{R}_{1}$. Significant differences between groups in GM and cortical lesions are only present in $\mathrm{R}_{1}$. No group differences are significant for AREX ${ }_{\text {Corr }}$ within cortical regions. All indices differ significantly between GM and WM within each group. ${ }^{*} p<0.05,{ }^{* *} p<0.01,{ }^{* \star *} p<0.001$.

(6-8, 66-68). Aspects of glutamate involvement in MS pathology include increased extracellular glutamate leading to excitotoxicity and neuronal and glial cell death, increased production of glutamate in macrophages and microglial cells in WM lesions (via elevation of glutaminase expression), and deficient glutamate reuptake by oligodendrocytes in MS WM $(7,69,70)$.

After MT correction, we did not find any significant differences in CEST indices (MTRasym $_{\text {Corr }}$, MTR RexCorr, $_{\text {, }}$ AREX $_{\text {Corr }}$ ) between NAGM or NAWM in patients with MS and the corresponding healthy GM or WM in healthy volunteers (Figure 5 and Supplementary Figure 1). The significant decrease in $\mathrm{R}_{1}$ observed in the NAGM of MS patients relative to healthy GM, and in GM and WM lesions relative to normalappearing and healthy tissues, support the importance of AREX calculation to remove confounding $\mathrm{R}_{1}$ effects from the observed CEST signal. After applying the AREX correction, we confirmed that $\mathrm{AREX}_{\text {Corr }}$ values did not correlate with $\mathrm{R}_{1}$ values in each tissue region (Supplementary Figure 3), indicating that $\mathrm{AREX}_{\mathrm{Corr}}$ is independent of $\mathrm{R}_{1}$ differences.
Our findings for $\mathrm{R}_{1}$ agree with prior studies showing lower $\mathrm{R}_{1}$ in $\mathrm{WM}$ lesions and in $\operatorname{NAGM}(46,71,72)$. Additionally, the decrease in GM:WM tissue contrast in CEST indices after removal of MT highlights the influence of MT effects on the quantified CEST signal (Figure 4) and corresponds with recent literature (45).

In a prior study, we found increased GluCEST contrast in the prefrontal cortex of RRMS patients compared to healthy volunteers; however, in that work, we implemented the conventional GluCEST quantification method and did not apply MT removal or $R_{1}$ corrections (16). In the current study, we observed non-significant trends toward increased GM AREX Corr $_{\text {}}$ in the prefrontal and parietal cortices of MS patients (Figure 5). Discrepancies in findings could be due to the influences of underlying myelin and $\mathrm{R}_{1}$ (71) changes that co-occur in MS pathology, and/or the use of a different cohort of MS patients. Reported glutamate abnormalities in MS GM have also varied in the MRS literature, and include no significant difference compared to controls (7), lower glutamate values in the cingulate 
and parietal cortices (8), and lower values in parietal and sensorimotor regions (68).

Although we did not detect differences in normal-appearing tissues in patients, we found that after removing the MT, spillover, and $\mathrm{R}_{1}$ contributions, $\mathrm{AREX}_{\mathrm{Corr}}$ in WM lesions was significantly

TABLE 3 | Measures of cognitive function.

\begin{tabular}{|c|c|c|}
\hline $\begin{array}{l}\text { Cognitive } \\
\text { assessment, mean } \pm \\
\text { SD }\end{array}$ & MS patients $(n=15)$ & Healthy volunteers $(n=20)$ \\
\hline $\begin{array}{l}\text { CVLT-II (trials 1-5 total } \\
\text { free recall) }\end{array}$ & $44.7 \pm 9.1^{\star \star \star}$ & $56.9 \pm 9.9$ \\
\hline $\begin{array}{l}\text { CVLT-II (long delay free } \\
\text { recall) }\end{array}$ & $9.2 \pm 3.2^{\star *}$ & $12.8 \pm 2.9$ \\
\hline SDMT (oral) & $52.7 \pm 12.2^{\star \star \star}$ & $65.5 \pm 8.1$ \\
\hline BVMT-R (total recall) & $24.3 \pm 6.1^{\dagger}$ & $27.4 \pm 4.1$ \\
\hline BVMT-R (delayed recall) & $8.8 \pm 2.6^{\dagger}$ * & $10.5 \pm 1.3$ \\
\hline $\begin{array}{l}\text { PASAT (2-second } \\
\text { version) }\end{array}$ & $33.2 \pm 9.2$ & $38.0 \pm 12.3$ \\
\hline $\begin{array}{l}\text { D-KEFS ST (total } \\
\text { correct, set } 1+\text { set 2) }\end{array}$ & $9.7 \pm 2.5$ & $10.9 \pm 2.6$ \\
\hline $\begin{array}{l}\text { Judgement of line } \\
\text { orientation }\end{array}$ & $25.2 \pm 4.3$ & $26.2 \pm 3.1$ \\
\hline $\begin{array}{l}\text { COWAT (total words, C } \\
+F+L)\end{array}$ & $34.0 \pm 7.1^{\star \star \star}$ & $45.6 \pm 9.0$ \\
\hline Simple reaction time & $295.9 \pm 46.1 \mathrm{~ms}^{\dagger}$ * & $266.4 \pm 32.4 \mathrm{~ms}$ \\
\hline Choice reaction time & $499.0 \pm 91.4 \mathrm{~ms}^{\dagger}$ & $452.8 \pm 85.1 \mathrm{~ms}$ \\
\hline
\end{tabular}

15 of the 20 patients with MS and 19 of the 20 healthy volunteers completed the Minimal Assessment of Cognitive Function in MS (MACFIMS) battery. The remaining 5 patients with MS completed a subset of the tests.

MS, multiple sclerosis; CVLT-II, California Verbal Learning Test, $2^{\text {nd }}$ edition; SDMT, Symbol Digit Modalities Test; BVMT-R, Brief Visuospatial Memory Test-Revised; PASAT, Paced Auditory Serial Addition Test; D-KEFS ST, Delis-Kaplan Executive Function System Sorting Test; COWAT, Controlled Oral Word Association Test.

${ }^{\star} p<0.05,{ }^{* \star} p<0.01,{ }^{* \star} p<0.001$ : Patients with MS differ significantly from healthy volunteers.

${ }^{\dagger}$ MS patients $n=20$. increased relative to NAWM and healthy WM (Figure 5 and Supplementary Figures 1, 2). To our knowledge, glutamateweighted CEST has not been evaluated in MS patients in studies other than our prior work, but APT-weighted CEST has been explored in MS. In a preliminary study of four MS patients, Dula et al. noted that APT-weighted CEST asymmetry within heterogeneous lesions could either increase or decrease relative to healthy WM (18). Another APT-weighted CEST study with a larger sample size of 27 relapsing-remitting and secondary progressive MS patients found that mean APT-weighted CEST signal intensity increased in MS lesions relative to healthy WM (17). Finally, an APT-weighted CEST study in patients with amnestic mild cognitive impairment (not MS) also found higher CEST signals in patients relative to healthy controls in several regions of the brain, including the GM and WM in the occipital and temporal lobes (73).

Although these prior CEST studies in MS and amnestic cognitive impairment quantify the CEST signal at $3.5 \mathrm{ppm}$ (attributed to amide protons on endogenous proteins and peptides), the glutamate-weighted CEST signal at $3.0 \mathrm{ppm}$ could also be influenced by amine protons on protein lysine residues as shown by in vitro experiments (14). We expect that glutamate is the primary contributor to the MT-corrected AREX Corr $_{\text {signal, }}$ since greater signal is observed in GM which has a higher glutamate concentration than WM. Our finding of increased glutamate-weighted $\mathrm{AREX}_{\text {Corr }}$ in WM lesions also corresponds with MR spectroscopy studies that found evidence of glutamate toxicity in MS patients via elevated glutamate in acute WM lesions (6) and in NAWM (7). Elevated glutamate in active WM lesions is likely caused by inflammatory infiltrates with excess glutamate released by activated leukocytes, macrophages, and microglial cells (6), along with impaired glutamate uptake by oligodendrocytes (70). Srinivasan et al. did not find elevated glutamate in chronic WM lesions (6), which may explain the greater inter-subject variability in glutamate-weighted AREX $_{\text {Corr }}$ signal of WM lesions relative to NAWM and healthy WM in our

\begin{tabular}{|c|c|c|c|c|c|c|c|c|c|c|c|c|c|}
\hline & \multicolumn{4}{|c|}{ R1 } & \multicolumn{9}{|c|}{ AREX at 3ppm } \\
\hline & WM & GM & WML & CXL & WM & GM & Prefrontal & Parietal & Occipital & Motor & Somato & WML & CXL \\
\hline EDSS & 0.12 & 0.16 & 0.23 & -0.03 & 0.26 & 0.19 & 0.24 & 0.17 & 0.60 & 0.02 & -0.05 & 0.06 & -0.10 \\
\hline Disease Duration & -0.10 & 0.06 & -0.20 & -0.14 & 0.06 & -0.08 & 0.20 & -0.14 & -0.22 & 0.06 & 0.08 & -0.08 & 0.40 \\
\hline SDMT (oral) & 0.52 & 0.37 & 0.66 & 0.41 & 0.55 & 0.36 & 0.26 & 0.30 & -0.17 & 0.15 & -0.11 & 0.66 & -0.03 \\
\hline BVMT-R Total Recall & 0.45 & 0.38 & -0.05 & -0.26 & 0.05 & -0.14 & 0.09 & -0.28 & -0.06 & -0.63 & -0.58 & -0.25 & -0.31 \\
\hline BVMT-R Delayed Recall & 0.26 & 0.21 & -0.16 & -0.31 & 0.04 & -0.10 & 0.07 & -0.18 & -0.07 & -0.54 & -0.41 & -0.21 & -0.26 \\
\hline CVLT-II T1to5 Free Recall & 0.43 & 0.21 & 0.19 & -0.25 & 0.15 & -0.05 & -0.24 & 0.01 & -0.51 & -0.14 & -0.21 & 0.29 & -0.39 \\
\hline CVLT-II Long Delay Free Recall & 0.52 & 0.27 & 0.55 & 0.07 & 0.15 & 0.00 & -0.13 & 0.03 & -0.43 & -0.16 & -0.29 & 0.27 & -0.44 \\
\hline PASAT (2-second) & 0.62 & 0.40 & 0.43 & 0.05 & -0.24 & -0.20 & -0.19 & -0.15 & -0.50 & -0.18 & -0.08 & 0.16 & -0.27 \\
\hline Simple Reaction Time & -0.03 & -0.03 & 0.05 & -0.54 & 0.24 & 0.16 & 0.27 & 0.17 & 0.23 & -0.03 & -0.39 & 0.39 & -0.28 \\
\hline Choice Reaction Time & -0.09 & -0.06 & -0.06 & -0.68 & 0.11 & -0.13 & -0.19 & -0.06 & -0.28 & -0.03 & 0.03 & 0.03 & 0.14 \\
\hline D-KEFS ST & 0.47 & 0.39 & 0.42 & 0.21 & 0.32 & 0.36 & 0.43 & 0.33 & -0.17 & 0.19 & 0.00 & 0.84 & 0.22 \\
\hline Judgement of Line Orientation & 0.45 & 0.51 & -0.16 & -0.06 & 0.25 & 0.00 & 0.05 & -0.04 & -0.36 & -0.05 & -0.02 & 0.25 & 0.10 \\
\hline COWAT & -0.15 & -0.38 & -0.34 & -0.07 & -0.17 & -0.29 & -0.44 & -0.18 & -0.28 & -0.21 & -0.04 & -0.25 & -0.11 \\
\hline
\end{tabular}

FIGURE 6 | Matrix of Spearman rho values for partial correlations between clinical and cognitive variables and mean $R_{1}$ and $A R E X$ values in patients with MS. Age, education, and sex were included as covariates. Correlations are highlighted in yellow if $p<0.05$. SDMT = Symbol Digit Modalities Test; BVMT-R = Brief Visuospatial Memory Test-Revised; CVLT-II = California Verbal Learning Test, 2nd edition; PASAT = Paced Auditory Serial Addition Test; D-KEFS ST = Delis-Kaplan Executive Function System Sorting Test; COWAT = Controlled Oral Word Association Test. 
study (Figure 5 and Supplementary Figures 1, 2), as we did not distinguish active from chronic lesions.

\section{AREX $X_{\text {corr }}$ and $R_{1}$ Values Are Associated With Disease Status and Cognitive Function}

The patient cohort in this study presented with mild cognitive impairment compared to the matched healthy cohort, with significantly lower scores on some of the tests administered and scores within normal limits on others (74-78). Lower performance for the patient cohort was observed in the domains of verbal and visuospatial learning and memory, information processing speed, verbal fluency, and cognitive flexibility; thus, we explored whether glutamate-sensitive CEST contrast and $\mathrm{R}_{1}$ were associated with cognitive function.

Despite multiple MRS studies showing increased glutamate in WM lesions and NAWM and decreased glutamate in NAGM, findings on the relationships between brain glutamate concentration and clinical outcomes in MS have varied. Azevedo et al. found that elevated glutamate alone in NAWM did not predict clinical measures of disability in a longitudinal study; however, an elevated glutamate/ $N$-acetylaspartate ratio in NAWM predicted a decline in brain volume and worsening clinical disability measures (MS Functional Composite, EDSS, and PASAT). Here, we did not find any significant correlations between AREX $_{\text {Corr }}$ in NAWM and measures of cognitive function or disease status (disease duration or EDSS), but we observed a significant positive correlation between AREX Corr in WM lesions and performance on the D-KEFS Sorting Test (Figure 6). The D-KEFS Sorting Test assesses executive function (e.g., categorization, abstraction, flexibility of thinking), a cognitive domain commonly affected in MS (79). Based on the significant increase in $\mathrm{AREX}_{\text {Corr }}$ in WM lesions relative to healthy WM, we might expect that higher $\mathrm{AREX}_{\text {Corr }}$ values would instead correspond with lower test scores. Although D-KEFS Sorting Test scores were lower in the patient group, the deficit was not significant and the patient group mean fell within normal limits (77). The observed association may also be confounded by not distinguishing active from chronic lesions.

Results from prior studies of GM glutamate levels relating to cognitive outcomes in MS are also mixed. An MRS study using large single-voxel measurements (ranging from 6.7 to $15.6 \mathrm{~mL}$ in volume) found that poor visuospatial memory performance was significantly associated with lower glutamate concentration in the hippocampus, thalamus, and cingulate cortex of MS patients but not in healthy controls (8), even after correcting for magnetization ratio. In contrast, another single-voxel MRS study initially found that parietal glutamate was a significant predictor of PASAT and SDMT performance; however, this relationship was no longer significant after adjusting for MT ratio, $\mathrm{N}$ acetylaspartate level, and normalized brain volume (markers of structural damage) (68). In our prior GluCEST study, we found that increased GluCEST contrast in the prefrontal cortex was significantly correlated with SDMT scores and Choice Reaction Time, both measures of information processing speed (16), although that analysis did not include corrections for MT and
$R_{1}$. In the current study, we found that glutamate-weighted AREX $_{\text {Corr }}$ in the occipital cortex was positively correlated with EDSS scores. Other significant relationships between GM AREX $_{\text {Corr }}$ and cognition included negative correlations between AREX $_{\text {Corr }}$ in the motor and somatosensory cortices and BVMT$\mathrm{R}$ scores, a measure of visuospatial learning and memory. The direction of these correlations also corresponds with our prior study in which higher glutamate in the cortex was associated with worse cognitive performance and increased disability (16). However, we would not typically expect these particular cortical regions to be associated with a test of visuospatial memory, and it is possible that this observed deficit is related to overall brain pathology rather than directly related to abnormal local glutamate levels. Additionally, while BVMT-R scores were lower in this patient cohort relative to the matched healthy cohort, the difference was only statistically significant in the delayed component of the test and the MS group mean was within normal limits for the BVMT-R (78).

We also examined correlations between $R_{1}$ and cognition in our patient cohort because $R_{1}$ measurements are notably sensitive to, but unspecific for, several pathological changes. Lower $\mathrm{R}_{1}$ in NAWM and in cortical lesions are documented features of MS $(71,72,80-83)$. Decreased $R_{1}$ can reflect increased water content, gliosis, and myelin and axonal loss, all known to occur diffusively in the NAWM. Damage to the WM in various tracts is known to be associated with cognitive impairment in several domains, mainly information processing, attention, and executive function (84-86). Cortical lesion burden is linked with disability and overall cognitive impairment, independently of subcortical WM damage $(87,88)$. Here, we found that higher $\mathrm{R}_{1}$ in the NAWM was significantly correlated with PASAT score, while higher $\mathrm{R}_{1}$ in cortical lesions was correlated with faster Choice Reaction Time. A high PASAT score and a faster Choice Reaction Time are both indices of good performance. Thus, detection of associations between worse cognitive scores and tissue alterations measured with $\mathrm{R}_{1}$ in different tissue compartments is an expected phenomenon and consistent with the expected associations between disease burden in the brain and cognition.

\section{Limitations and Conclusions}

Some limitations of this study include the modest sample size of MS patients with complete cognitive battery data, which limited our ability to detect independent effects of different CEST MRIderived variables given the number of covariates (e.g., age, sex, education), and the mild cognitive impairment status of these patients (several test scores within normal limits). Additionally, we did not correct for atrophy in our analyses because atrophy is a global process in the brain in MS, and the CEST data represented a $10 \mathrm{~mm}$-thick slice. A coarse slice thickness was chosen to increase the signal-to-noise ratio (SNR) of CEST which is typically a low-SNR acquisition, but this low throughplane resolution could cause some partial volume contamination, especially for small lesions. Future studies could consider 3D acquisitions or other faster imaging methods to improve both inplane and through-plane resolution. We did not separate active lesions from chronic lesions since no gadolinium contrast was 
used in this study, but future studies of glutamate in MS lesions may benefit from a separation of lesion type/status since different pathological processes could result in increased or decreased glutamate within an individual patient or patient cohort. In terms of glutamate-weighted CEST signal quantification, we chose to use $-3.0 \mathrm{ppm}$ as the reference frequency for the signal at $3.0 \mathrm{ppm}$, which could potentially be affected by rNOE effects as described above despite the sequence being optimized for sensitivity to glutamate CEST effects. We used a relatively simple two-pool Lorentzian model to fit the broad MT baseline in our CEST data and it performed well, but future studies could also employ higher spectral resolution for z-spectra and acquire offset frequencies outside the typical CEST window of -5.0-5.0 ppm to take advantage of other strategies for fitting CEST z-spectrum components $(30,61)$ and modeling the macromolecular MT effects that confound CEST quantification (22). Future work may also benefit from using an 8-channel parallel transmit system at 7.0 $\mathrm{T}$ to improve the homogeneity of the $\mathrm{B}_{1}+$ field for CEST saturation pulses. Finally, we did not correct for multiple comparisons in our analyses to avoid overcorrecting in this exploratory study. With a larger sample size and correction for multiple correlations, the strength of the associations observed in the current cohort may change. In Figure 5, there were 38 $t$-tests performed for all variables combined; with a Bonferroni correction, the significance level would be $p<0.0013$ and the differences observed between WM lesions and healthy WM and between WM and GM within each group remain significant $(p<$ 0.001). The correlations observed in Figure 6 would not remain significant after a correction for multiple comparisons given the current sample size and number of variables.

Despite the limitations of this study, our results highlight the utility of glutamate-weighted CEST at ultrahigh field as a metabolic imaging technique to probe the pathology underlying clinical symptoms, including cognitive impairment, in neurological diseases such as MS, and we demonstrated the importance of isolating the glutamate-weighted CEST signal from confounding factors such as changes in $R_{1}$ and macromolecular MT effects that occur simultaneously in demyelinating, inflammatory diseases.

\section{DATA AVAILABILITY STATEMENT}

The raw data supporting the conclusions of this article will be made available by the authors, without undue reservation.

\section{ETHICS STATEMENT}

The studies involving human participants were reviewed and approved by Vanderbilt University Institutional Review Board.

\section{REFERENCES}

1. DeLuca GC, Yates RL, Beale H, Morrow SA. Cognitive impairment in multiple sclerosis: clinical, radiologic and pathologic insights. Brain Pathol. (2015) 25:79-98. doi: 10.1111/bpa. 12220
The patients/participants provided their written informed consent to participate in this study.

\section{AUTHOR CONTRIBUTIONS}

KO'G, FB, HW, and SSm designed the study and interpreted the results. KO'G, SSa, QO, BB, and HF performed the experiments. KO'G, SSa, SC, AC, and BR contributed to image processing and data analysis. RL, RD, BR, and SSm provided technical advice. $\mathrm{FB}, \mathrm{AS}, \mathrm{SM}$, JN, and KY performed and confirmed segmentation of lesions. KO'G, AC, and SSm wrote the paper. All authors contributed constructively to the manuscript.

\section{FUNDING}

Research reported in this publication was supported in part by funding from the National Institutes of Health (NINDS award numbers F32NS101788, R01NS109114, and R21NS116434-01A1, NCATS Vanderbilt CTSA award number UL1TR000445, NCATS award number KL2TR002245, and NIBIB award number K01EB030039), the U.S. Department of Defense (award number W81XWH-13-1-0073), the National Multiple Sclerosis Society (award numbers RG-1501-02840 and RG-1901-33190), the Conrad Hilton Foundation, the Veterans Health Administration (award number I01CX002160-01A1) and the VUMC Faculty Research Scholars program. The content is solely the responsibility of the authors and does not necessarily represent the official views of the National Institutes of Health. The authors declare that $\mathrm{FB}$ received funding from Biogen Idec. The funder was not involved in the study design, collection, analysis, interpretation of data, the writing of this article or the decision to submit it for publication.

\section{ACKNOWLEDGMENTS}

The authors thank the VUIIS technologists (Christopher Thompson, Clair Jones, Leslie McIntosh, and Kristen GeorgeDurett), VUIIS Center for Human Imaging, Charles Nockowski, Dr. Allen Newton, Dr. Adrienne Dula, and Dr. Samantha By. This work was conducted in part using the resources of the Center for Computational Imaging at Vanderbilt University Institute of Imaging Science, Nashville, TN.

\section{SUPPLEMENTARY MATERIAL}

The Supplementary Material for this article can be found online at: https://www.frontiersin.org/articles/10.3389/fneur. 2022.764690/full\#supplementary-material
2. Rocca MA, Amato MP, De Stefano N, Enzinger C, Geurts JJ, Penner IK, et al. Clinical and imaging assessment of cognitive dysfunction in multiple sclerosis. Lancet Neurol. (2015) 14:302-17. doi: 10.1016/S1474-4422(14)70250-9

3. Kostic M, Zivkovic N, Stojanovic I. Multiple sclerosis and glutamate excitotoxicity. Rev Neurosci. (2013) 24:7188. doi: 10.1515/revneuro-2012-0062 
4. Macrez R, Stys PK, Vivien D, Lipton SA, Docagne F. Mechanisms of glutamate toxicity in multiple sclerosis: biomarker and therapeutic opportunities. Lancet Neurol. (2016) 15:1089-102. doi: 10.1016/S1474-4422(16)3 0165-X

5. Swanberg KM, Landheer K, Pitt D, Juchem C. Quantifying the metabolic signature of multiple sclerosis by in vivo proton magnetic resonance spectroscopy: current challenges and future outlook in the translation from proton signal to diagnostic biomarker. Front Neurol. (2019) 10:1173. doi: 10.3389/fneur.2019.01173

6. Srinivasan R, Sailasuta N, Hurd R, Nelson S, Pelletier D. Evidence of elevated glutamate in multiple sclerosis using magnetic resonance spectroscopy at $3 \mathrm{~T}$. Brain. (2005) 128:1016-25. doi: 10.1093/brain/awh467

7. Azevedo CJ, Kornak J, Chu P, Sampat M, Okuda DT, Cree BA, et al. In vivo evidence of glutamate toxicity in multiple sclerosis. Ann Neurol. (2014) 76:269-78. doi: 10.1002/ana.24202

8. Muhlert N, Atzori M, De Vita E, Thomas DL, Samson RS, WheelerKingshott CA, et al. Memory in multiple sclerosis is linked to glutamate concentration in grey matter regions. J Neurol Neurosurg Psychiatry. (2014) 85:833-9. doi: 10.1136/jnnp-2013-306662

9. van Zijl PC, Yadav NN. Chemical exchange saturation transfer (CEST): what is in a name and what isn't? Magn Reson Med. (2011) 65:92748. doi: $10.1002 / \mathrm{mrm} .22761$

10. Zhou J, van Zijl PCM. Chemical exchange saturation transfer imaging and spectroscopy. Prog Nucl Magn Reson Spectrosc. (2006) 48:10936. doi: 10.1016/j.pnmrs.2006.01.001

11. Zhou J, Wilson DA, Sun PZ, Klaus JA, Van Zijl PC. Quantitative description of proton exchange processes between water and endogenous and exogenous agents for WEX, CEST, and APT experiments. Magn Reson Med. (2004) 51:945-52. doi: 10.1002/mrm.20048

12. Jones KM, Pollard AC, Pagel MD. Clinical applications of chemical exchange saturation transfer (CEST) MRI. J Magn Reson Imaging. (2018) 47:1127. doi: 10.1002/jmri.25838

13. Cai K, Haris M, Singh A, Kogan F, Greenberg JH, Hariharan H, et al. Magnetic resonance imaging of glutamate. Nat Med. (2012) 18:3026. doi: $10.1038 / \mathrm{nm} .2615$

14. Cui J, Zhang XY, Xie J, Gochberg DF, Zu Z. Towards the molecular origin of glutamate CEST (GluCEST) imaging in rat brain. Magn Reson Med. (2020) 83:1405-17. doi: 10.1002/mrm.28021

15. Haris M, Nath K, Cai K, Singh A, Crescenzi R, Kogan F, et al. Imaging of glutamate neurotransmitter alterations in Alzheimer's disease. NMR Biomed. (2013) 26:386-91. doi: 10.1002/nbm.2875

16. O'Grady KP, Dula AN, Lyttle BD, Thompson LM, Conrad BN, Box BA, et al. Glutamate-sensitive imaging and evaluation of cognitive impairment in multiple sclerosis. Mult Scler. (2019) 25:1580-92. doi: 10.1177/1352458518799583

17. Sartoretti E, Sartoretti T, Wyss M, Becker AS, Schwenk A, van Smoorenburg L, et al. Amide proton transfer weighted imaging shows differences in multiple sclerosis lesions and white matter hyperintensities of presumed vascular origin. Front Neurol. (2019) 10:1307. doi: 10.3389/fneur.2019.01307

18. Dula AN, Asche EM, Landman BA, Welch EB, Pawate S, Sriram S, et al. Development of chemical exchange saturation transfer at 7 T. Magn Reson Med. (2011) 66:831-8. doi: 10.1002/mrm.22862

19. Thomas AM, Xu J, Calabresi PA, van Zijl PCM, Bulte JWM. Monitoring diffuse injury during disease progression in experimental autoimmune encephalomyelitis with on resonance variable delay multiple pulse (onVDMP) CEST MRI. Neuroimage. (2020) 204:116245. doi: 10.1016/j.neuroimage.2019.116245

20. By S, Barry RL, Smith AK, Lyttle BD, Box BA, Bagnato FR, et al. Amide proton transfer CEST of the cervical spinal cord in multiple sclerosis patients at $3 \mathrm{~T}$. Magn Reson Med. (2018) 79:806-14. doi: 10.1002/mrm.26736

21. Lee DW, Heo H, Woo CW, Woo DC, Kim JK, Kim KW, et al. Temporal changes in in vivo glutamate signal during demyelination and remyelination in the corpus callosum: a glutamate-weighted chemical exchange saturation transfer imaging study. Int J Mol Sci. (2020) 21:9468. doi: 10.3390/ijms21249468

22. Smith AK, Ray KJ, Larkin JR, Craig M, Smith SA, Chappell MA. Does the magnetization transfer effect bias chemical exchange saturation transfer effects? Quantifying chemical exchange saturation transfer in the presence of magnetization transfer. Magn Reson Med. (2020) 84:135975. doi: 10.1002/mrm.28212

23. Mallik S, Samson RS, Wheeler-Kingshott CA, Miller DH. Imaging outcomes for trials of remyelination in multiple sclerosis. J Neurol Neurosurg Psychiatry. (2014) 85:1396-404. doi: 10.1136/jnnp-2014-307650

24. Vavasour IM, Laule C, Li DK, Traboulsee AL, MacKay AL. Is the magnetization transfer ratio a marker for myelin in multiple sclerosis? J Magn Reson Imaging. (2011) 33:713-8. doi: 10.1002/jmri.22441

25. Li AX, Hudson RH, Barrett JW, Jones CK, Pasternak SH, Bartha R. Four-pool modeling of proton exchange processes in biological systems in the presence of MRI-paramagnetic chemical exchange saturation transfer (PARACEST) agents. Magn Reson Med. (2008) 60:1197-206. doi: 10.1002/mrm.21752

26. Desmond KL, Moosvi F, Stanisz GJ. Mapping of amide, amine, and aliphatic peaks in the CEST spectra of murine xenografts at 7 T. Magn Reson Med. (2014) 71:1841-53. doi: 10.1002/mrm.24822

27. Zaiss M, Schmitt B, Bachert P. Quantitative separation of CEST effect from magnetization transfer and spillover effects by Lorentzian-line-fit analysis of Z-spectra. J Magn Reson. (2011) 211:149-55. doi: 10.1016/j.jmr.2011.05.001

28. Zaiss M, Xu J, Goerke S, Khan IS, Singer RJ, Gore JC, et al. Inverse Z-spectrum analysis for spillover-, MT-, and T1 -corrected steady-state pulsed CESTMRI-application to pH-weighted MRI of acute stroke. NMR Biomed. (2014) 27:240-52. doi: 10.1002/nbm.3054

29. Zaiss M, Bachert P. Exchange-dependent relaxation in the rotating frame for slow and intermediate exchange - modeling off-resonant spin-lock and chemical exchange saturation transfer. NMR Biomed. (2013) 26:50718. doi: $10.1002 / \mathrm{nbm} .2887$

30. Zaiss M, Windschuh J, Paech D, Meissner JE, Burth S, Schmitt B, et al. Relaxation-compensated CEST-MRI of the human brain at 7T: Unbiased insight into NOE and amide signal changes in human glioblastoma. Neuroimage. (2015) 112:180-8. doi: 10.1016/j.neuroimage.2015.02.040

31. Zhang XY, Wang F, Li H, Xu J, Gochberg DF, Gore JC, et al. CEST imaging of fast exchanging amine pools with corrections for competing effects at $9.4 \mathrm{~T}$. NMR Biomed. (2017) 30:e3715. doi: 10.1002/nbm.3715

32. Polman CH, Reingold SC, Banwell B, Clanet M, Cohen JA, Filippi M, et al. Diagnostic criteria for multiple sclerosis: 2010 revisions to the McDonald criteria. Ann Neurol. (2011) 69:292-302. doi: 10.1002/ana.22366

33. Benedict RH, Fischer JS, Archibald CJ, Arnett PA, Beatty WW, Bobholz J, et al. Minimal neuropsychological assessment of MS patients: a consensus approach. Clin Neuropsychol. (2002) 16:381-97. doi: 10.1076/clin.16.3.381.13859

34. Reicker LI, Tombaugh TN, Walker L, Freedman MS. Reaction time: an alternative method for assessing the effects of multiple sclerosis on information processing speed. Arch Clin Neuropsychol. (2007) 22:65564. doi: 10.1016/j.acn.2007.04.008

35. Marques JP, Kober T, Krueger G, van der Zwaag W, Van de Moortele PF, Gruetter R. MP2RAGE, a self bias-field corrected sequence for improved segmentation and T1-mapping at high field. Neuroimage. (2010) 49:127181. doi: 10.1016/j.neuroimage.2009.10.002

36. Visser F, Zwanenburg JJ, Hoogduin JM, Luijten PR. High-resolution magnetization-prepared 3D-FLAIR imaging at 7.0 Tesla. Magn Reson Med. (2010) 64:194-202. doi: 10.1002/mrm.22397

37. Yarnykh VL. Actual flip-angle imaging in the pulsed steady state: a method for rapid three-dimensional mapping of the transmitted radiofrequency field. Magn Reson Med. (2007) 57:192-200. doi: 10.1002/mrm.21120

38. Kim M, Gillen J, Landman BA, Zhou J, van Zijl PC. Water saturation shift referencing (WASSR) for chemical exchange saturation transfer (CEST) experiments. Magn Reson Med. (2009) 61:1441-50. doi: 10.1002/mrm.21873

39. McKeithan LJ, Lyttle BD, Box BA, O'Grady KP, Dortch RD, Conrad BN, et al. 7T quantitative magnetization transfer (qMT) of cortical gray matter in multiple sclerosis correlates with cognitive impairment. Neuroimage. (2019) 203:116190. doi: 10.1016/j.neuroimage.2019.116190

40. Dortch RD, Moore J, Li K, Jankiewicz M, Gochberg DF, Hirtle JA, et al. Quantitative magnetization transfer imaging of human brain at $7 \mathrm{~T}$. Neuroimage. (2013) 64:640-9. doi: 10.1016/j.neuroimage.2012.08.047

41. Dortch RD, Bagnato F, Gochberg DF, Gore JC, Smith SA. Optimization of selective inversion recovery magnetization transfer imaging for macromolecular content mapping in the human brain. Magn Reson Med. (2018) 80:1824-35. doi: 10.1002/mrm.27174 
42. Jenkinson M, Bannister P, Brady M, Smith S. Improved optimization for the robust and accurate linear registration and motion correction of brain images. Neuroimage. (2002) 17:825-41. doi: 10.1006/nimg.2002.1132

43. Jenkinson $M$, Smith S. A global optimisation method for robust affine registration of brain images. Med Image Anal. (2001) 5:143-56. doi: 10.1016/S1361-8415(01)00036-6

44. Jones CK, Huang A, Xu J, Edden RA, Schar M, Hua J, et al. Nuclear overhauser enhancement (NOE) imaging in the human brain at 7T. Neuroimage. (2013) 77:114-24. doi: 10.1016/j.neuroimage.2013.03.047

45. Debnath A, Hariharan H, Nanga RPR, Reddy R, Singh A. Glutamateweighted CEST contrast after removal of magnetization transfer effect in human brain and rat brain with tumor. Mol Imaging Biol. (2020) 22:1087101. doi: 10.1007/s11307-019-01465-9

46. Bagnato F, Hametner S, Franco G, Pawate S, Sriram S, Lassmann H, et al. Selective inversion recovery quantitative magnetization transfer brain MRI at 7T: clinical and postmortem validation in multiple sclerosis. J Neuroimaging. (2018) 28:380-8. doi: 10.1111/jon.12511

47. Schmierer K, Scaravilli F, Altmann DR, Barker GJ, Miller DH. Magnetization transfer ratio and myelin in postmortem multiple sclerosis brain. Ann Neurol. (2004) 56:407-15. doi: 10.1002/ana.20202

48. Janve VA, Zu Z, Yao SY, Li K, Zhang FL, Wilson KJ, et al. The radial diffusivity and magnetization transfer pool size ratio are sensitive markers for demyelination in a rat model of type III multiple sclerosis (MS) lesions. Neuroimage. (2013) 74:298-305. doi: 10.1016/j.neuroimage.2013.02.034

49. Mulkern RV, Williams ML. The general solution to the bloch equation with constant rf and relaxation terms: application to saturation and slice selection. Med Phys. (1993) 20:5-13. doi: 10.1118/1.597063

50. Hua J, Jones CK, Blakeley J, Smith SA, van Zijl PC, Zhou J. Quantitative description of the asymmetry in magnetization transfer effects around the water resonance in the human brain. Magn Reson Med. (2007) 58:78693. doi: $10.1002 / \mathrm{mrm} .21387$

51. McMahon MT, Gilad AA, Zhou J, Sun PZ, Bulte JW, van Zijl PC. Quantifying exchange rates in chemical exchange saturation transfer agents using the saturation time and saturation power dependencies of the magnetization transfer effect on the magnetic resonance imaging signal (QUEST and QUESP): Ph calibration for poly-L-lysine and a starburst dendrimer. Magn Reson Med. (2006) 55:836-47. doi: 10.1002/mrm.20818

52. Singh A, Debnath A, Cai K, Bagga P, Haris M, Hariharan H, et al. Evaluating the feasibility of creatine-weighted CEST MRI in human brain at $7 \mathrm{~T}$ using a Z-spectral fitting approach. NMR Biomed. (2019) 32:e4176. doi: 10.1002/nbm.4176

53. O’Brien KR, Kober T, Hagmann P, Maeder P, Marques J, Lazeyras F, et al. Robust T1-weighted structural brain imaging and morphometry at $7 \mathrm{~T}$ using MP2RAGE. PLoS ONE. (2014) 9:e99676. doi: 10.1371/journal.pone.00 99676

54. Huo Y, Xu Z, Xiong Y, Aboud K, Parvathaneni P, Bao S, et al. 3D whole brain segmentation using spatially localized atlas network tiles. Neuroimage. (2019) 194:105-19. doi: 10.1016/j.neuroimage.2019.03.041

55. Huo Y, Xu Z, Aboud K, Parvathaneni P, Bao S, Bermudez C, et al. Spatially localized atlas network tiles enables $3 \mathrm{D}$ whole brain segmentation. In: International Conference on Medical Image Computing and Computer Assisted Intervention - MICCAI 2018. Granada: Springer (2018).

56. Hadel S, Wirth C, Rapp M, Gallinat J, Schubert F. Effects of age and sex on the concentrations of glutamate and glutamine in the human brain. J Magn Reson Imaging. (2013) 38:1480-7. doi: 10.1002/jmri.24123

57. Kaiser LG, Schuff N, Cashdollar N, Weiner MW. Age-related glutamate and glutamine concentration changes in normal human brain: 1H MR spectroscopy study at 4 T. Neurobiol Aging. (2005) 26:665-72. doi: 10.1016/j.neurobiolaging.2004.07.001

58. O'Gorman RL, Michels L, Edden RA, Murdoch JB, Martin E. In vivo detection of GABA and glutamate with MEGA-PRESS: reproducibility and gender effects. J Magn Reson Imaging. (2011) 33:1262-7. doi: 10.1002/jmri.22520

59. Crescenzi R, DeBrosse C, Nanga RP, Reddy S, Haris M, Hariharan H, et al. In vivo measurement of glutamate loss is associated with synapse loss in a mouse model of tauopathy. Neuroimage. (2014) 101:18592. doi: 10.1016/j.neuroimage.2014.06.067

60. Davis KA, Nanga RP, Das S, Chen SH, Hadar PN, Pollard JR, et al. Glutamate imaging (GluCEST) lateralizes epileptic foci in nonlesional temporal lobe epilepsy. Sci Transl Med. (2015) 7:309ra161. doi: 10.1126/scitranslmed.aaa7095

61. Windschuh J, Zaiss M, Meissner JE, Paech D, Radbruch A, Ladd ME, et al. Correction of B1-inhomogeneities for relaxation-compensated CEST imaging at 7 T. NMR Biomed. (2015) 28:529-37. doi: 10.1002/nbm.3283

62. Liu D, Zhou J, Xue R, Zuo Z, An J, Wang DJ. Quantitative characterization of nuclear overhauser enhancement and amide proton transfer effects in the human brain at 7 tesla. Magn Reson Med. (2013) 70:107081. doi: $10.1002 / \mathrm{mrm} .24560$

63. Pitt D, Werner P, Raine CS. Glutamate excitotoxicity in a model of multiple sclerosis. Nat Med. (2000) 6:67-70. doi: 10.1038/71555

64. Basso AS, Frenkel D, Quintana FJ, Costa-Pinto FA, Petrovic-Stojkovic S, Puckett L, et al. Reversal of axonal loss and disability in a mouse model of progressive multiple sclerosis. J Clin Invest. (2008) 118:153243. doi: 10.1172/JCI33464

65. Lee DW, Woo DC, Heo H, Kim KW, Kim JK, Lee DH. Signal alterations of glutamate-weighted chemical exchange saturation transfer MRI in lysophosphatidylcholine-induced demyelination in the rat brain. Brain Res Bull. (2020) 164:334-8. doi: 10.1016/j.brainresbull.2020.09.004

66. Matute C, Domercq M, Sanchez-Gomez MV. Glutamate-mediated glial injury: mechanisms and clinical importance. Glia. (2006) 53:212-24. doi: 10.1002/glia.20275

67. Chard DT, Griffin CM, McLean MA, Kapeller P, Kapoor R, Thompson AJ, et al. Brain metabolite changes in cortical grey and normal-appearing white matter in clinically early relapsing-remitting multiple sclerosis. Brain. (2002) 125:2342-52. doi: 10.1093/brain/awf240

68. Nantes JC, Proulx S, Zhong J, Holmes SA, Narayanan S, Brown RA, et al. GABA and glutamate levels correlate with MTR and clinical disability: insights from multiple sclerosis. Neuroimage. (2017) 157:70515. doi: 10.1016/j.neuroimage.2017.01.033

69. Werner P, Pitt D, Raine CS. Multiple sclerosis: altered glutamate homeostasis in lesions correlates with oligodendrocyte and axonal damage. Ann Neurol. (2001) 50:169-80. doi: 10.1002/ana.1077

70. Pitt D, Nagelmeier IE, Wilson HC, Raine CS. Glutamate uptake by oligodendrocytes: Implications for excitotoxicity in multiple sclerosis. Neurology. (2003) 61:1113-20. doi: 10.1212/01.WNL.0000090564.88719.37

71. Steenwijk MD, Vrenken H, Jonkman LE, Daams M, Geurts JJ, Barkhof F, et al. High-resolution T1-relaxation time mapping displays subtle, clinically relevant, gray matter damage in long-standing multiple sclerosis. Mult Scler. (2016) 22:1279-88. doi: 10.1177/1352458515615953

72. Vrenken H, Geurts JJ, Knol DL, van Dijk LN, Dattola V, Jasperse B, et al. Whole-brain T1 mapping in multiple sclerosis: global changes of normal-appearing gray and white matter. Radiology. (2006) 240:81120. doi: 10.1148/radiol.2403050569

73. Zhang Z, Zhang C, Yao J, Chen $\mathrm{X}$, Gao $\mathrm{F}$, Jiang $\mathrm{S}$, et al. Protein-based amide proton transfer-weighted MR imaging of amnestic mild cognitive impairment. Neuroimage Clin. (2020) 25:102153. doi: 10.1016/j.nicl.2019.102153

74. Delis DC, Kramer JH, Kaplan E, Ober BA. California Verbal Learning Test Second Edition-Adult Version. Bloomington, MN: Pearson, Inc (2000).

75. Benton AL, Sivan A, Hamsher K, Varney N, Spreen O. Contributions to Neuropsychology Assessment: A Clinical Manual. 2nd ed. New York: Oxford University Press (1994).

76. Ruff RM, Light RH, Parker SB, Levin HS. Benton controlled oral word association test: reliability and updated norms. Arch Clin Neuropsychol. (1996) 11:329-38. doi: 10.1093/arclin/11.4.329

77. Delis DC, Kaplan E, Kramer JH. Delis-Kaplan Executive Function System. San Antonio, TX: Pearson, Inc. (2001).

78. Benedict RHB. Brief Visuospatial Memory Test-Revised. Lutz, FL: Psychological Assessment Resources, Inc (1997).

79. Riccardi A, Puthenparampil M, Rinaldi F, Ermani M, Perini P, Gallo P, et al. Failure identifies multiple sclerosis patients with worse objective and self-perceived physical and cognitive disability. Front Psychol. (2019) 10:49. doi: 10.3389/fpsyg.2019.00049

80. Stevenson VL, Parker GJ, Barker GJ, Birnie K, Tofts PS, Miller DH, et al. Variations in $\mathrm{T} 1$ and $\mathrm{T} 2$ relaxation times of normal appearing white matter and lesions in multiple sclerosis. J Neurol Sci. (2000) 178:817. doi: 10.1016/S0022-510X(00)00339-7 
81. Parry A, Clare S, Jenkinson M, Smith S, Palace J, Matthews PM. White matter and lesion $\mathrm{T} 1$ relaxation times increase in parallel and correlate with disability in multiple sclerosis. J Neurol. (2002) 249:1279-86. doi: 10.1007/s00415-0020837-7

82. Kober T, Granziera C, Ribes D, Browaeys P, Schluep M, Meuli R, et al. MP2RAGE multiple sclerosis magnetic resonance imaging at 3 T. Invest Radiol. (2012) 47:346-52. doi: 10.1097/RLI.0b013e31824 $600 \mathrm{e} 9$

83. Beck ES, Sati $P$, Sethi V, Kober T, Dewey B, Bhargava $P$, et al. Improved visualization of cortical lesions in multiple sclerosis using $7 \mathrm{~T}$ MP2RAGE. AJNR Am J Neuroradiol. (2018) 39:459-66. doi: 10.3174/ajnr. A5534

84. Dineen RA, Vilisaar J, Hlinka J, Bradshaw CM, Morgan PS, Constantinescu CS, et al. Disconnection as a mechanism for cognitive dysfunction in multiple sclerosis. Brain. (2009) 132:239-49. doi: 10.1093/brain/a wn275

85. Filippi M, Rocca MA, Benedict RH, DeLuca J, Geurts JJ, Rombouts SA, et al. The contribution of MRI in assessing cognitive impairment in multiple sclerosis. Neurology. (2010) 75:2121-8. doi: 10.1212/WNL.0b013e31820 0d768

86. Preziosa P, Rocca MA, Pagani E, Stromillo ML, Enzinger C, Gallo A, et al. Structural MRI correlates of cognitive impairment in patients with multiple sclerosis: a multicenter study. Hum Brain Mapp. (2016) 37:1627-44. doi: 10.1002/hb m. 23125
87. Roosendaal SD, Moraal B, Pouwels PJ, Vrenken H, Castelijns JA, Barkhof $\mathrm{F}$, et al. Accumulation of cortical lesions in MS: relation with cognitive impairment. Mult Scler. (2009) 15:708-14. doi: 10.1177/1352458509102907

88. Calabrese M, Filippi M, Gallo P. Cortical lesions in multiple sclerosis. Nat Rev Neurol. (2010) 6:438-44. doi: 10.1038/nrneurol.2010.93

Conflict of Interest: The authors declare that the research was conducted in the absence of any commercial or financial relationships that could be construed as a potential conflict of interest.

Publisher's Note: All claims expressed in this article are solely those of the authors and do not necessarily represent those of their affiliated organizations, or those of the publisher, the editors and the reviewers. Any product that may be evaluated in this article, or claim that may be made by its manufacturer, is not guaranteed or endorsed by the publisher.

Copyright () 2022 O'Grady, Satish, Owen, Box, Bagnato, Combes, Cook, Westervelt, Feiler, Lawless, Sarma, Malone, Ndolo, Yoon, Dortch, Rogers and Smith. This is an open-access article distributed under the terms of the Creative Commons Attribution License (CC BY). The use, distribution or reproduction in other forums is permitted, provided the original author(s) and the copyright owner(s) are credited and that the original publication in this journal is cited, in accordance with accepted academic practice. No use, distribution or reproduction is permitted which does not comply with these terms. 